\title{
LES/CMC Modelling of a Gas Turbine Model Combustor with Quick Fuel Mixing
}

\author{
Huangwei Zhang ${ }^{1}$ and Epaminondas Mastorakos ${ }^{2}$ \\ ${ }^{1}$ Department of Mechanical Engineering, National University of Singapore, 9 Engineering Drive 1, \\ Singapore 117576, Republic of Singapore \\ ${ }^{2}$ Department of Engineering, University of Cambridge, Cambridge CB2 1PZ, United Kingdom
}

Type of article: full-length article (original research paper)

Author: $\quad$ Huangwei Zhang (corresponding author)

Assistant Professor

Department of Mechanical Engineering

Faculty of Engineering, National University of Singapore

9 Engineering Drive 1, Singapore 117576

Republic of Singapore

Tel: +65 6516 2557, Fax: +65 67791459

Email: huangwei.zhang@nus.edu.sg

Epaminondas Mastorakos

Professor

Department of Engineering

University of Cambridge

Trumpington Street, Cambridge CB2 1PZ, UK

Tel: +44 12233 32690, Fax: +44 1223332690

Email: em257@eng.cam.ac.uk

Paper submitted to : $\quad$ Flow, Turbulence and Combustion on June $25^{\text {th }}, 2018$

Revision submitted to: Flow, Turbulence and Combustion on September $14^{\text {th }}, 2018$ Accepted by Flow, Turbulence and Combustion on September 21st, 2018 


\title{
LES/CMC Modelling of a Gas Turbine Model Combustor with Quick Fuel Mixing
}

\author{
Huangwei Zhang $^{1 *}$ and Epaminondas Mastorakos ${ }^{2}$ \\ ${ }^{1}$ Department of Mechanical Engineering, National University of Singapore, 9 Engineering Drive 1, \\ Singapore 117576, Republic of Singapore \\ ${ }^{2}$ Department of Engineering, University of Cambridge, Cambridge CB2 1PZ, United Kingdom
}

\begin{abstract}
The first-order, single-conditioned sub-grid Conditional Moment Closure (CMC) model for Large Eddy Simulation (LES) is applied to simulate a globally lean swirling methane flame in a gas turbine model combustor that has been studied experimentally. The time-averaged velocity, mixture fraction, temperature, major species and $\mathrm{OH}$ mass fractions, and the heat release rate are predicted well for most locations. A transient lift-off and flashback of the flame root due to localized extinction near the burner exit is observed that is qualitatively consistent with the experimental measurements. The timeaveraged temperature is over-predicted very close to the fuel injection point, while it is accurately reproduced downstream. Comparisons of instantaneous conditionally-filtered temperature in mixture fraction space shows that the LES/CMC reproduces the large scatter of the experimental data points in temperature-mixture fraction plane that span the full range unburnt to fully burnt, but to a smaller extent, suggesting a minor under-prediction of local extinction or the inaccuracy of the present first-order, coarse-grid $\mathrm{CMC}$ formulation to capture locally premixed flame propagation behaviour. Periodic variation of the heat release rate is observed with a frequency close to that of the measured Precessing Vortex Core (PVC). In general, the current LES/CMC model captures most features of this high-mixingrate nominally non-premixed swirl flame in a gas turbine model combustor in agreement with
\end{abstract}

\footnotetext{
* Corresponding author. E-mail: huangwei.zhang@nus.edu.sg. Tel: +65 65162557.
} 
experimental measurements.

Keywords: gas turbine model combustor, fuel-lean combustion, swirl-stabilized flames, large eddy simulation, conditional moment closure 


\section{Introduction}

The Large-Eddy Simulation (LES) / Conditional Moment Closure (CMC) combustion model [1] has reproduced many features of non-premixed combustion with significant finite-rate kinetics effects. For example, the degree of local extinction in piloted jet flames [2,3] and in the Sydney methane [4] and the Cambridge methane [5] non-premixed swirl flames, the lift-off statistics in spray flames [6], and even the full blow-off curve (i.e. the air flow rate at blow-off for a range of fuel flow rates) in swirling flames [7] have been captured well. There has also been extensive validation against spray flame databases [8,9] and for diesel engine autoignition, flame stabilization, and pollutant emissions [10-12]. The common feature across these flames is that there are significant mixture fraction fluctuations in the region of the measurements that have been used for validation. However, many gas turbine flames currently aim to have very quick mixing, perhaps almost reaching premixed (stratified or homogeneous) mixture conditions in parts of the reaction zone even if the fuel and air are injected separately. The extent to which the conventional LES/CMC model conditioning on single mixture fraction [2-9] can capture such local behaviors has not been explored yet.

A flame with methane injection from numerous small holes placed in an annular arrangement between two swirling air streams has been studied at DLR [13,14], aiming to reproduce a wellcharacterized flame configuration relevant to lean-burn gas turbine combustors. The pointwise measurements of temperature versus mixture fraction $(\xi)$ showed that the majority of the data points lie along conventional non-premixed flame distributions in $\eta$-space, but also that, close to the injection point and inside the Inner Recirculation Zone (IRZ), there are transitions in fluid composition between completely unburnt to completely burnt, for the same mixture fraction within the nominally flammable range $[13,14]$. This suggests locally premixed flame behavior. One of the measured flames (i.e. Flame A, following the naming in Refs. [13,14]) has been simulated in Refs. [15-18], showing different levels of agreement. Nevertheless, the accuracy of LES/CMC for flames with such characteristics has not been 
quantified yet.

The objective of this work is to quantitatively assess the accuracy of LES/CMC for modelling a globally fuel-lean flame with separate fuel/air injection and fast mixing. The target flame is the methane/air flame (Flame C) close to lean blow-off $[13,14,19]$. To the best of our knowledge, this is the first numerical simulation for Flame C in the DLR swirl burner with detailed chemical mechanism and advanced combustion model. Models and flame information will be given in Section 2, while the results and discussion will be presented in Section 3, followed by the conclusions in the final Section.

\section{Mathematical models and flow configuration}

\subsection{LES and CMC models}

The LES equations for filtered continuity, momentum and mixture fraction can be derived through filtering the respective instantaneous equations. The anisotropic part of the sub-grid stress tensor is closed with the constant Smagorinsky model. The filtered scalar dissipation rate $\widetilde{N}$ is calculated through [20]

$$
\widetilde{N}=\widetilde{N}_{r e s}+\widetilde{N}_{s g s}=\underbrace{D \nabla \tilde{\xi} \cdot \nabla \tilde{\xi}}_{\widetilde{N}_{r e s}}+\underbrace{\frac{c_{N}}{2} \frac{\mu_{t}}{\bar{\rho}_{t}^{2}} \widetilde{\xi}^{\prime \prime 2}}_{\widetilde{N}_{s g s}},
$$

where $\widetilde{N}_{r e s}$ and $\widetilde{N}_{s g s}$ are the filtered and sub-grid scale scalar dissipation, respectively. In Eq. (1), $c_{N}$ $=42[2]$ is used. $\bar{\rho}$ is the filtered density and $\Delta$ is the filter width calculated as $\Delta=V_{L E S}^{1 / 3} \cdot V_{L E S}$ denotes the volume of LES cells. $D$ represents the mass diffusivity and is calculated through $D=\mu / \bar{\rho} S c$ with Schmidt number $S c=1.0 . \mu$ is the molecular dynamic viscosity. $\tilde{\xi}$ and $\tilde{\xi}^{\pi 2}$ are filtered mixture fraction and its variance, respectively. $\widetilde{\xi^{\prime 2}}$ is modelled as (assuming $c_{v}=0.1[21]$ )

$$
\widetilde{\xi^{\prime \prime 2}}=c_{v} \Delta^{2} \nabla \tilde{\xi} \cdot \nabla \tilde{\xi}
$$

The three-dimensional governing equations for the conditionally filtered mass fractions of $\alpha$-th species, i.e. $Q_{\alpha} \equiv \widetilde{Y_{\alpha}} \eta$, are formulated as $[1,3,5,4,7,22]$ 


$$
\frac{\partial Q_{\alpha}}{\partial t}+\nabla \cdot\left(\widetilde{\mathbf{U} \eta} Q_{\alpha}\right)=Q_{\alpha} \nabla \cdot \widetilde{\mathbf{U} \eta} \eta \widetilde{N \mid \eta} \frac{\partial^{2} Q_{\alpha}}{\partial^{2} \eta}+\widetilde{\omega_{\alpha} \mid \eta}+e_{f}
$$

in which $\eta$ is the sample space variable for $\xi$. $\widetilde{\mathbf{U}} \eta, \widetilde{N \mid \eta}$, and $\widetilde{\omega_{\alpha} \mid \eta}$ are the conditionally filtered velocity, scalar dissipation rate, and reaction rates of the $\alpha$-th species, respectively. The assumption $\widetilde{\mathbf{U} \mid \eta} \approx \widetilde{\mathbf{U}}$ is adopted here. For $\widetilde{N \mid \eta}$, the Amplitude Mapping Closure model [23] is used, i.e. $\widetilde{N \mid \eta}=N_{0} G(\eta)$, where $N_{0}=\widetilde{N} / \int_{0}^{1} \widetilde{P}(\eta) G(\eta) d \eta$ and $\mathrm{G}(\eta)=\exp \left(-2\left[\operatorname{erf}^{-1}(2 \eta-1)\right]^{2}\right)$. The filtered scalar dissipation rate $\widetilde{N}$ is calculated with Eq. (1). The Filtered probability Density Function (FDF) $\widetilde{P}(\eta)$ is assumed to have a beta-function shape calculated with $\tilde{\xi}$ and $\widetilde{\xi^{\prime 2}}$. The first order CMC model is applied for estimating conditional chemical source terms in Eq. (3) such that $\widetilde{\omega_{\alpha} \mid \eta} \approx \omega_{\alpha}\left(Q_{1}, \ldots Q_{n}, Q_{T}\right)$, where $n$ is the number of species and $Q_{T} \equiv \widetilde{T \mid \eta}$ is the conditionally filtered temperature. The sub-grid scale diffusion $e_{f}$ is modelled as $e_{f}=\nabla \cdot\left(D_{t} \nabla Q_{\alpha}\right)$ [22]. Here $D_{t}$ is the turbulent diffusivity, estimated from $D_{t}=\mu_{t} / \bar{\rho} S c_{t}$ with the turbulent Schmidt number $S c_{t}=0.7 . \overline{\rho \mid \eta}$ is the conditionally filtered density. The conditionally filtered total enthalpy $Q_{h} \equiv \widetilde{h \eta}$ is also solved, which has the identical form as Eq. (2), but without the conditional chemical source term $\widetilde{\omega_{\alpha} \mid \eta}$. Additional source term for $Q_{h}$ equation is included, accounting for the convective heat loss from the combustor walls [7]. The unconditional reactive scalars $\tilde{f}$ (e.g. $\tilde{T}$ and $\tilde{Y}_{\alpha}$ ) can be convoluted from their respective conditional profiles, i.e. $\tilde{f}=\int_{0}^{l} \widetilde{f} \widetilde{P} \widetilde{P}(\eta) d \eta$.

\subsection{Flame information}

The simulated gas turbine model combustor is shown in Fig. 1(a) and was experimentally studied in DLR $[13,19,14]$. The combustion chamber has a square cross-section of $0.085 \times 0.085 \mathrm{~m}^{2}$ and a height of $0.11 \mathrm{~m}$. There is a conical exhaust gas stack connected to the chamber with diameter of $0.04 \mathrm{~m}$ and length of $0.05 \mathrm{~m}$. The dry air flow is supplied from an upstream plenum with an inner diameter of 0.079 $\mathrm{m}$ and a height of $0.065 \mathrm{~m}$. Inner and outer guided-vane swirlers are used to generate the co-swirling air 
streams leading to the chamber, through the concentric central (inner) and annular (outer) contoured nozzles, respectively. The swirlers have 8 and 12 channels respectively for central and annular nozzles. The diameter of the central nozzle is $0.015 \mathrm{~m}$, while the inner and outer diameters for the annular nozzle are $0.017 \mathrm{~m}$ and $0.025 \mathrm{~m}$, respectively. The non-swirling fuel injectors have 72 channels with size of $0.0005 \times 0.0005 \mathrm{~m}^{2}$ and lies as a ring between the central and annular nozzles. They are $0.0045 \mathrm{~m}$ upstream of the burner exit.

In this work, Flame $\mathrm{C}$ (following the naming convention in Refs. [13,14]) is simulated. This flame is close to lean blow-off (the corresponding lean extinction limit is $\Phi_{\text {glob,ext }}=0.53$ ) with thermal power $P_{t h}=7.6 \mathrm{~kW}$ and global equivalence ratio $\Phi_{g l o b}=0.55$ (corresponding mixture fraction is $\xi_{g l o b}=0.031$ ). Flame dynamics like lift-off, flashback and localized extinction have been observed [19]. The fuel is $100 \%$ methane and both air and fuel are at $298 \mathrm{~K}$. The stoichiometric mixture fraction is $\xi_{s t}=0.055$. The mass flow rates are respectively $0.00015 \mathrm{~kg} / \mathrm{s}$ and $0.00468 \mathrm{~kg} / \mathrm{s}$. The ratio of the mass flows from the common plenum to central and annular nozzles are $\sigma \approx 1.5$, which is estimated from the experimental measurements [13]. Numerical tests were made through decoupling the mass flow rates of two swirlers in a non-reacting case (with the same volume flow rates as in Flame A [14] and replacement of methane in fuel jets with air jets) by See [24] and Ramaekers et al. [25], to investigate the effects of $\sigma$ on the flow pattern in the chamber. Their results demonstrate that the flow characteristics are strongly affected by this ratio [24]. Their treatment about mass flow splitting can, to some degree, simplify the simulation (e.g. reduce both geometry and mesh scale), but undoubtedly introduce new issues (e.g. specification of the turbulence at the artificial inlets after part geometry is removed, and/or the uncertainties about the adjusted $\sigma$ to match the experimental flow patterns). As such, in the current numerical simulations, two swirlers in the computational domain are included. Furthermore, the gross swirl number is about 0.55 , calculated based on the velocity profiles immediately above the nozzle exit without considering the local pressure effect [13]. 

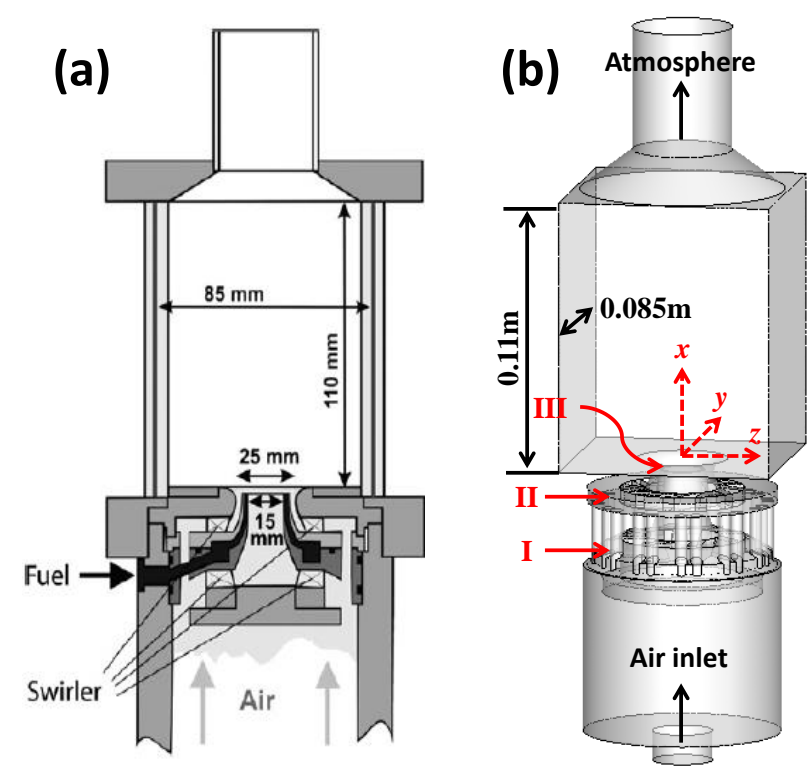

Fig. 1 Dual swirler burner $[13,19,14]$ : (a) schematic and (b) computational domain. I: inner swirler; II: outer swirler; III: fuel injectors.

\subsection{Numerical implementation}

Mixture fraction space is discretised by 51 nodes with refinement around $\xi_{s t}$ In physical space, the Cartesian coordinate origin lies at the center of the burner exit (i.e. longitudinally $0.0045 \mathrm{~m}$ downstream of fuel inlet channels, see Fig. 1b), with $x$ being streamwise coordinate and $y / z$ being spanwise coordinates. Both LES and CMC computational domains (see Fig. 1b) include the combustor and a downstream hemispherical far-field (not shown here). Approximately 22 million tetrahedral LES cells are generated, while the number of the CMC cells is about 169,000. Local mesh refinement (nominal cell size ranges from $0.0001 \mathrm{~m}$ to $0.0002 \mathrm{~m}$ ) is made for the swirlers, central and annular nozzles, connection between the chamber and nozzle, as well as the flame regions in the upstream of the chamber. In particular, since there is rounded expansion at the outer nozzle exit (cf. Fig. 1a) that may produce considerable flow separation and further influence the Outer Recirculation Zone (ORZ), the local mesh there is also refined to $0.0001 \mathrm{~m}$.

For CMC boundaries in mixture fraction space, $\eta=0$ corresponds to air and $\eta=1$ to fuel, both at 
298 K. For LES boundaries, at all the walls, non-slip condition is applied for the velocities and zero gradient for the mixture fraction. The wall temperature $T_{W}$ is assumed to be $298 \mathrm{~K}$. This is different from the measured value (i.e. $600 \mathrm{~K}$ [14]), but its influence on the combustion in the chamber is expected to be negligible. Zero pressure gradient and Dirichlet velocity and mixture fraction conditions are enforced for the air and fuel inlets which are at the burner exit plane while fixed total atmospheric pressure for the far-field boundaries.

About the CMC boundaries in physical space, the inert mixing solutions are specified in the air and fuel inlets as well as at walls, while zero gradient condition is enforced at the far-field outlet. All the CMC cells are initialized with the fully burning distributions of $Q_{\alpha}$.

Both LES and CMC governing equations are discretized using a finite volume method. For LES, an OpenFOAM solver with low Ma number assumption is used. The PISO algorithm is used for the velocity-pressure coupling and a first-order implicit Euler scheme for time marching. The time step $\Delta t$ is $2 \times 10^{-6} \mathrm{~s}$, to ensure the maximum $\mathrm{CFL}<0.75$. For $\mathrm{CMC}$, an in-house solver is adopted for solving Eq. (3) and is interfaced with LES. Full operator splitting is used for the terms in Eq. (3). The conditional chemistry terms $\widetilde{\omega_{\alpha} \mid \eta}$ are calculated by the ODE solver VODPK [26]. The micro-mixing term in Eq. (3) is calculated with TDMA (i.e. Tri-Diagonal Matrix Algorithm) method. Time integration for CMC equations is performed by first-order Euler scheme for the conditional convection, dilatation and subgrid diffusion terms. The first-order upwind and second-order central differencing schemes are used for conditional convection and sub-grid diffusion terms in $\mathrm{CMC}$ equations, while about the conditional dilatation term the linear interpolation is used for calculating the velocities at the CMC faces. The ARM2 mechanism (19 species and 15 reactions) by Sung et al. [27] is used. 160 processors are applied for the LES/CMC computations. Approximately $0.003 \mathrm{~s}$ of physical time can be achieved with 24-hour wall clock time. The statistics are collected for $0.06 \mathrm{~s}$, after the initial field effects are minimized. The mean axial velocity at the nozzle exit is about $10 \mathrm{~m} / \mathrm{s}$ (as shown in Fig. 5) and the combustor streamwise length 
is $0.11 \mathrm{~m}$, which gives $0.011 \mathrm{~s}$ as the flow-through time. Therefore, the sampling duration of $0.06 \mathrm{~s}$ corresponds to about 5.5 flow-through timescales. Strictly speaking, the proper way to examine statistical convergence is based on the autocorrelation timescale, not the flow through-time. However, as the autocorrelation timescale varies significantly across the combustor, the same level of statistical convergence cannot be achieved for all locations. Taking $5 \mathrm{~mm}$ as an estimate of typical integral lengthscale at the shear layer and using Taylor's hypothesis, the integral autocorrelation time is about 0.5 ms, which implies that the sampling time includes about 22 autocorrelation timescales. Estimating further the turbulence intensity as about 20-40\% (Figs. 5 and 7), the relative statistical error on the reported velocity statistics is about $4-8 \%$.

\section{Results and discussion}

\subsection{General flame features}

The filtered and time-averaged axial velocities are shown in Figs. 2(a) and 2(b). Two recirculation zones are evident: the long IRZ and relatively compact ORZ, similar to other confined swirl burners $[5,7]$. The IRZ's longitudinal distance (marked with $I$ in Fig. 2b) and maximum spanwise width (II in Fig. 2b) are about 0.07 and $0.02 \mathrm{~m}$, respectively, which agree fairly well with the experimental data (i.e. 0.071 and $0.019 \mathrm{~m} \mathrm{[13]).} \mathrm{In} \mathrm{addition,} \mathrm{the} \mathrm{IRZ} \mathrm{also} \mathrm{extends} \mathrm{slightly} \mathrm{into} \mathrm{the} \mathrm{central} \mathrm{nozzle.} \mathrm{The} \mathrm{angle} \mathrm{of} \mathrm{the}$ peak time-averaged axial velocity with respect to the centerline is about $25^{\circ}$, close to $26^{\circ}$ reported by

Weigand et al. [14]. Also, transient flow separation near the rounded expansion at the outer nozzle exit can be observed from the LES/CMC simulations. Based on Figs. 2(c) and 2(d), the swirling motion decays quickly after about one-fourth of the chamber length, and becomes strong again close to the stack due to the contraction. The computed ratio of mean mass flow rate for central and annular nozzles is about 1.38 , close to the experimental estimate of $\sigma \approx 1.5[13]$.

Figures 2(e) and 2(f) show that the mixture fraction is well below the stoichiometric value, i.e. 
$\xi_{s t}=0.055$, in most of the combustion chamber, indicating the fuel-lean operating condition in Flame C. The lean flammability limit $\left(\xi_{\text {lean }}=0.0465\right.$; see isoline in Figs. $2 \mathrm{i}$ and $\left.2 \mathrm{j}\right)$ is confined to the region immediately downstream of the fuel inlets. In most of IRZ, $\xi$ is close to the well-mixed value of $\xi_{g l o b}=$ 0.031. This is due to the strong turbulence created by the shear between the annular jets and the IRZ and ORZ and the swirling motion. High values of the filtered temperature appears along the IRZ upstream boundaries (see Fig. 2g), where the flame stabilizes, while further downstream, it becomes more uniform, consistent with the experimental data (see Fig. 15 in Ref. [13]).

Comparisons of computed and measured flame shapes are made in Fig. 3. Firstly, for the $\mathrm{OH}$ mass fraction, the current LES/CMC reproduces the following main features: (1) OH lies in a narrow region inclined $45^{\circ}$ with respect to the centerline, with no considerable $\mathrm{OH}$ present in ORZ; (2) the OH mass fraction is generally low $(\sim 0.001)$, except of the centerline close to $x=0.01 \mathrm{~m}$. However, this localized high $\mathrm{OH}$ concentration in Fig. 3(a) is not observed from the measured results in Fig. 3(b). Secondly, the time-averaged heat release distribution is consistent with the averaged $\mathrm{OH}^{*}$ chemiluminescence image (see Figs. 3c and 3d). Nevertheless, discrepancies also exist. The simulated peak heat release rate location is close to the central air nozzle, while it is found further downstream in the measurements. These differences will be further discussed in Section 3.2.

Figure 4 shows instantaneous contours of filtered $\mathrm{OH}$ and experimental OH-PLIF and the general $\mathrm{OH}$ distribution are similar. In the LES, the flame root (lowest locus with finite $\mathrm{OH}$ in Fig. 4a) moves back and forth with time, and even occasionally flashes back into the central nozzle (not shown here). Close to it, there is some local extinction (see Fig. 4a), but its role for complete blowout cannot be further interpreted here due to the limited duration of our simulation. Further discussion about transient flame dynamics for Flame C will be made in Section 3.4. 


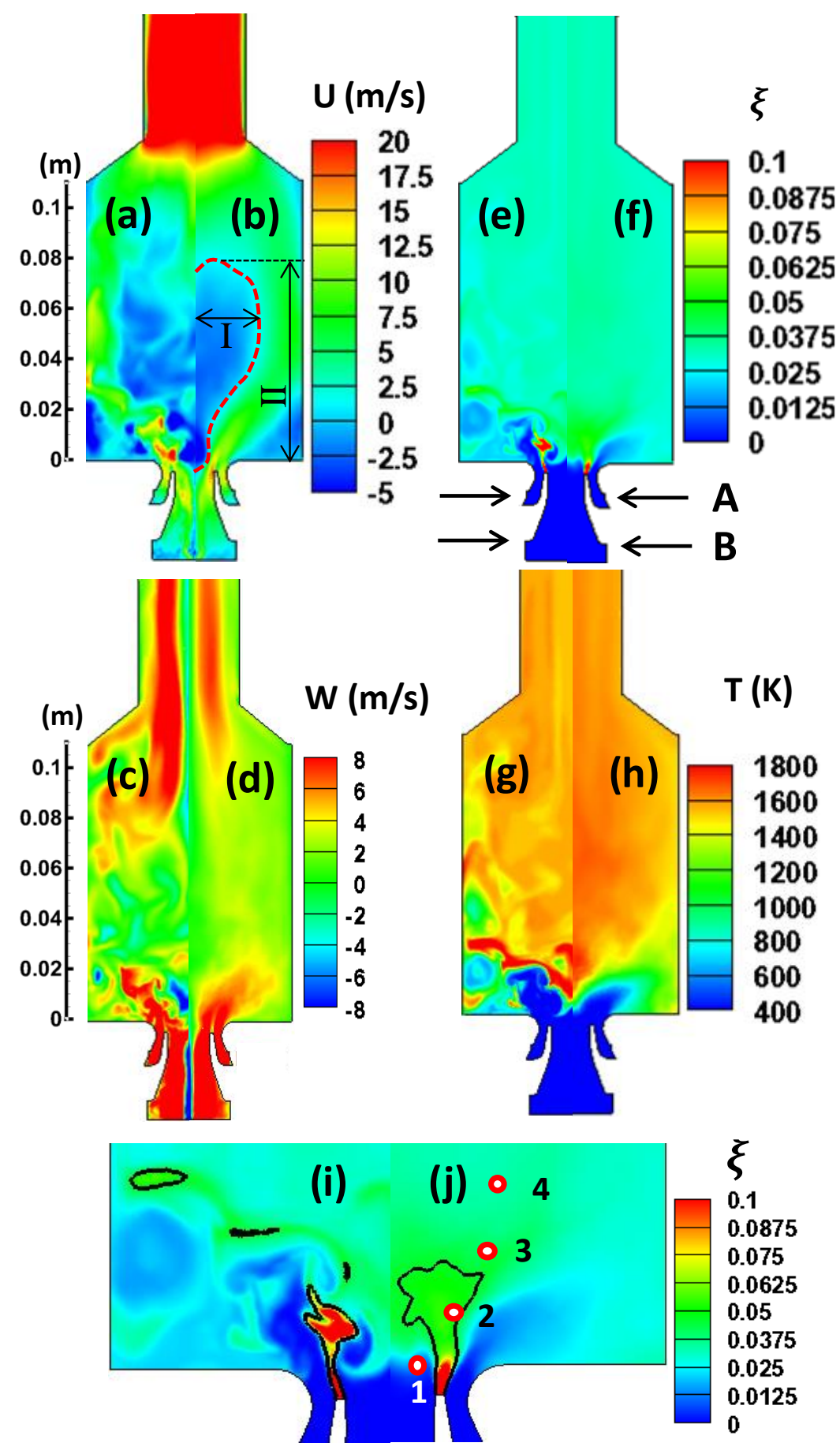

Fig. 2 Contours of axial velocity (a,b), swirl velocity $(c, d)$, mixture fraction (e,f) and temperature $(\mathrm{g}, \mathrm{h})$. Mixture fraction fields near fuel injectors are shown in (i) and (j). Left subfigures: instantaneous filtered fields; right sub-figures: time-averaged fields. $A$ : outer swirling air; $B$ : inner swirling air. $I \approx 20 \mathrm{~mm}$ and $I I \approx 70 \mathrm{~mm}$. Isolines in (a) and (b): zero axial velocity; isolines in (i) and (j): mixture fraction corresponding to methane lean flammability limit $\left(\xi_{\text {lean }}=0.0465\right)$. The circles are the probed locations for PSD analysis in Fig. 17. 


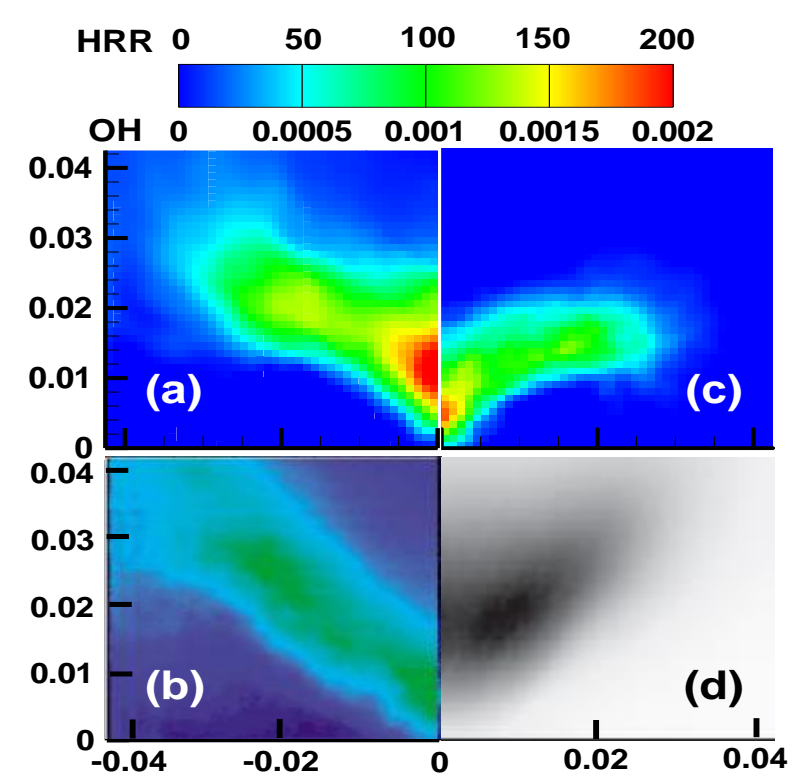

Fig. 3 Comparisons of (a) time-averaged $\mathrm{OH}$ mass fraction and (b) heat release rate (in $\mathrm{MJ} / \mathrm{m}^{3} \mathrm{~s}$ ) respectively with (c) time-averaged OH-PLIF [13] and (d) $\mathrm{OH}^{*}$ chemiluminescence [19]. Color bar only for (a) and (c).
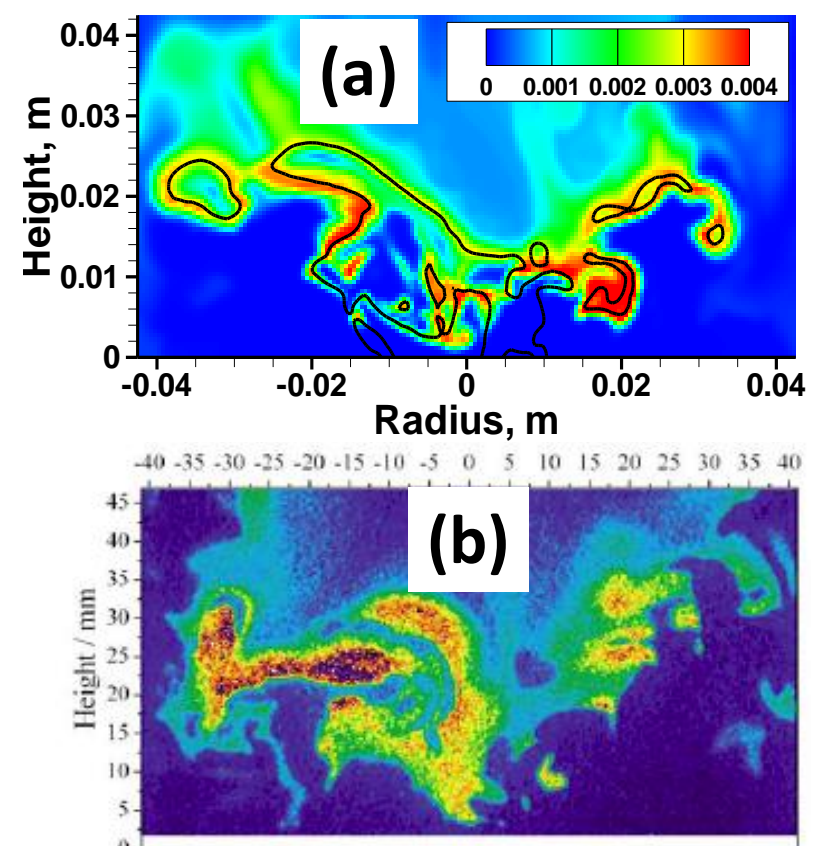

Fig. 4 Instantaneous (a) filtered OH mass fraction and (b) OH-PLIF [13]. Isolines in (a): $\xi_{s t}=$ 0.055 . 


\subsection{Velocity and scalar fields in physical space}

The simulated time-averaged axial and swirl velocities agree very well with experiment (see Figs. $5-6)$. At $x=0.001 \mathrm{~m}$, the recirculation intensity (quantified by magnitude of mean axial velocity) is overpredicted, while at $x=0.01$ and $0.02 \mathrm{~m}$, it is slightly under-predicted. The solid-body rotation motion immediately downstream of the central and annular air nozzles (i.e. $-0.025 \mathrm{~m} \leq x \leq 0.025 \mathrm{~m}$ at $x=$ $0.001 \mathrm{~m}$ ) is accurately reproduced. However, swirl velocities in the ORZ for $x=0.005 \mathrm{~m}$ and $0.01 \mathrm{~m}$ are slightly over-predicted. In addition, the solid-body rotation motion close to the stack (see Figs. 6h and 6i) is also accurately computed. Therefore we conclude that our LES/CMC simulations correctly capture the main time-averaged axial and swirl velocity fields from the burn exit $(x=1 \mathrm{~mm})$ to chamber outlet $(x=$ $100 \mathrm{~mm})$, based on the comparisons with the experimental data in Figs. 5 and 6.

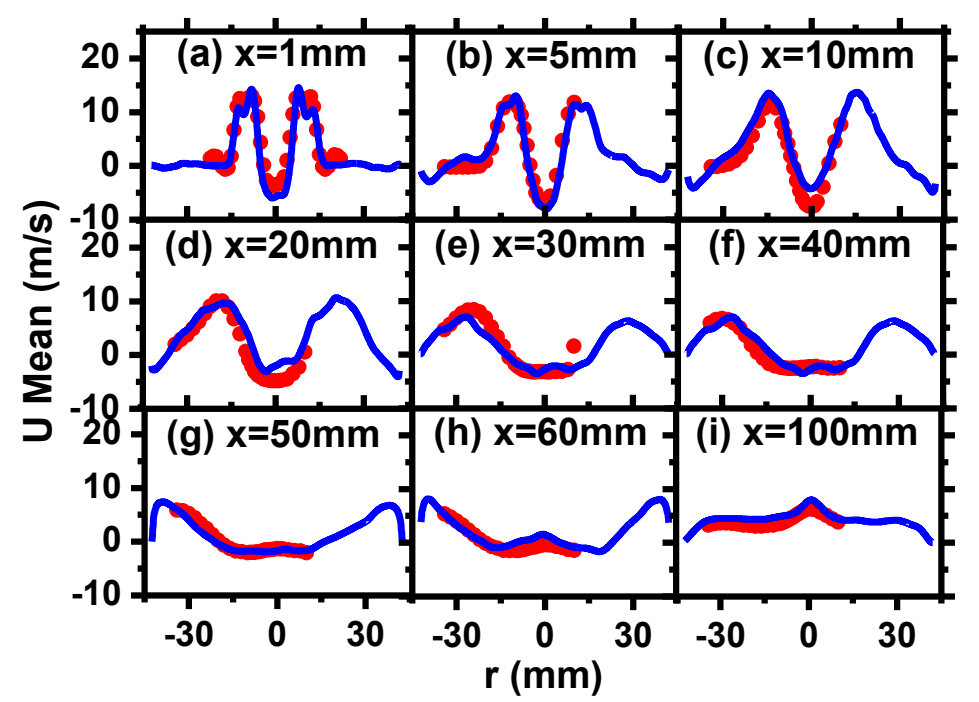

Fig. 5 Radial profiles of mean axial velocity. Lines: LES/CMC; symbols: experimental data from Ref. [13]. 


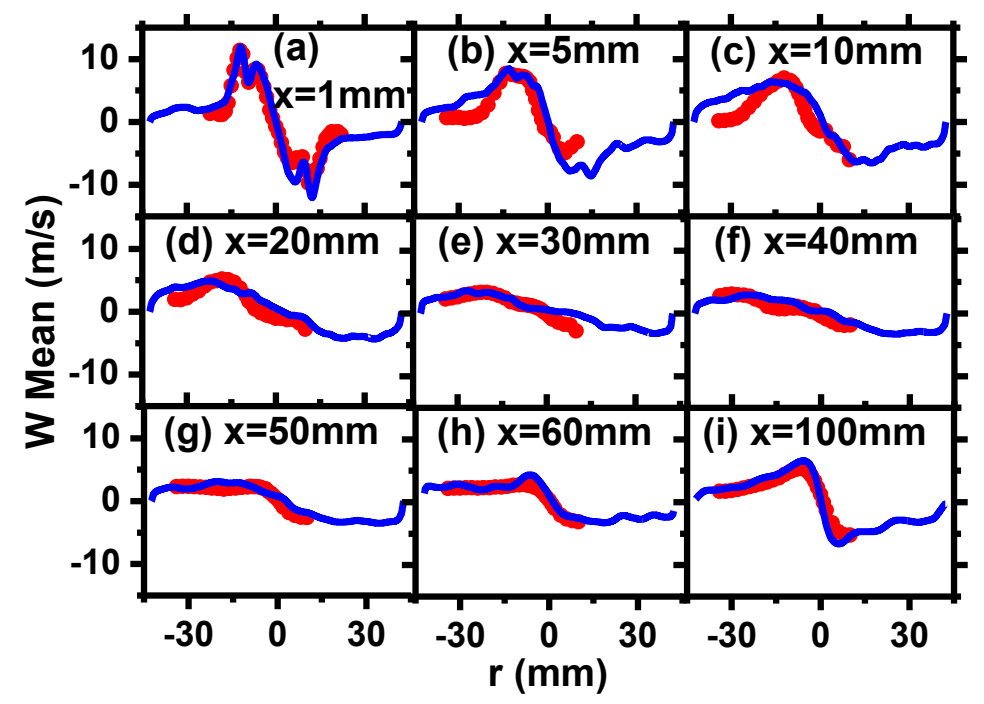

Fig. 6 Radial profiles of mean swirl velocity. Lines: LES/CMC; symbols: experimental data from Ref. [13].

Figures 7 and 8 show the various radial profiles of RMS axial and swirl velocities computed from the filtered axial and swirl velocities. The RMS for both velocity components at $x=0.001 \mathrm{~m}$ are well captured, as shown in Figs. 7(a) and 8(a). This location (i.e. $x=0.001 \mathrm{~m}$ ) is very close to the inlet of the chamber. Therefore, results obtained here, together with the mean velocity results in Figs. 5(a) and 5(b), indicate that the velocity profiles at the inlet to the combustion chamber are accurately predicted. For downstream regions in the chamber, the values and locations of the computed peak RMS velocities agree well with the experimental results. The pronounced over-predictions (about $15 \%$ for the peak values) are observable for $|r|>0.015 \mathrm{~m}$ (actually $r<-0.015 \mathrm{~m}$, due to the unavailability of the experimental data for $r>0.015 \mathrm{~m}$ ). This corresponds to the ORZ where the turbulent flow fields are highly transient, affected by the recirculating flows and vortex shedding from the shear layer between the swirling air stream and ORZ. More downstream (e.g. $x=0.05 \mathrm{~m}$ in Figs. $7 \mathrm{f}$ and $8 \mathrm{f}$ ), very good agreement is achieved between the simulation and measurement data. Overall, with the velocity statistics in Figs. 5-8, one can see that the LES/CMC accurately predicts the statistics of the velocity field of Flame $\mathrm{C}$ in this gas turbine model combustor. 


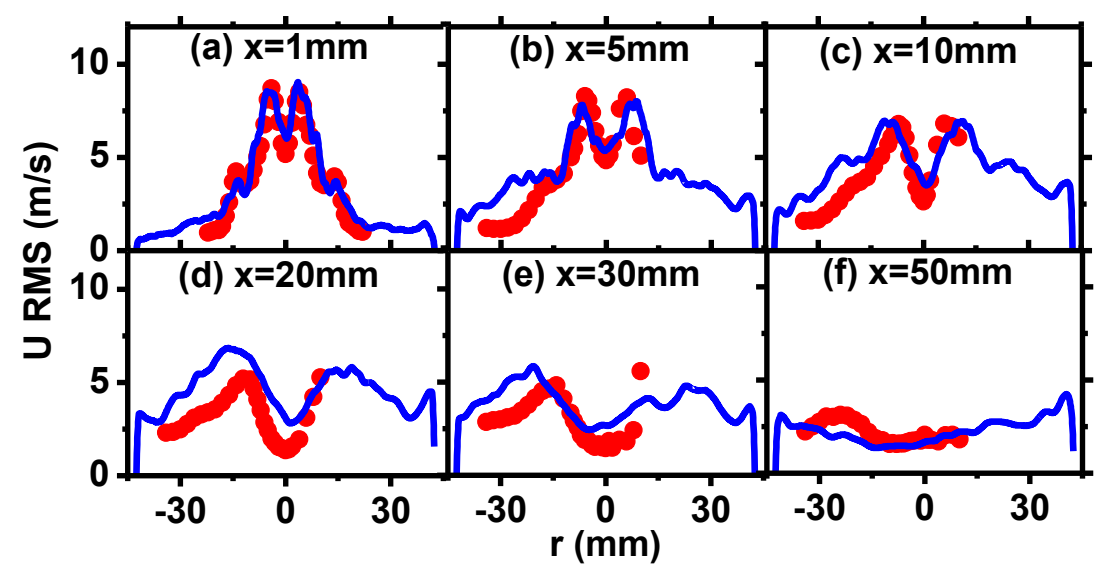

Fig. 7 Radial profiles of axial RMS velocity. Lines: LES/CMC; symbols: experimental data from Ref. [13].

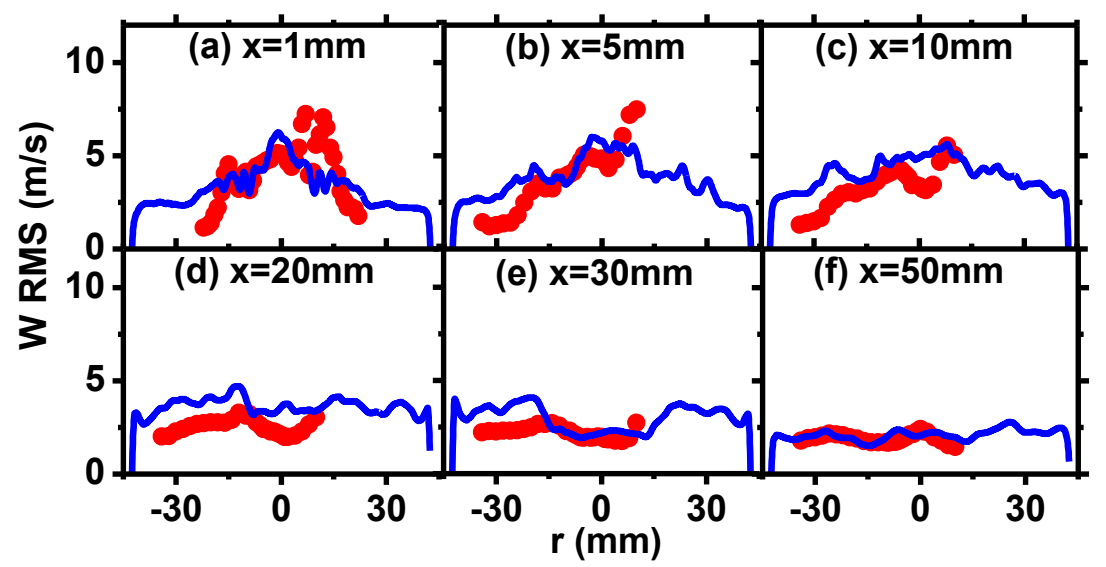

Fig. 8 Radial profiles of swirl RMS velocity. Lines: LES/CMC; symbols: experimental data from Ref. [13].

The mean mixture fraction is also very well computed as shown in Fig. 9, except for $x=0.005 \mathrm{~m}$ where a slight under-prediction of the peak of the mean $\xi$, with about $10 \%$ lower than the experimental counterparts, is evident. The mixture fraction is below $\xi_{s t}=0.055$ and also below fuel-lean flammability limit $\xi_{\text {lean }}=0.0465$ for most of the shown locations, and approaches $\xi_{\text {glob }}=0.031$ downstream (say $x \geq 0.02 \mathrm{~m}$ ), as also demonstrated in Figs. 2(e) and 2(f). Weigand et al. also mentioned that, for Flame C, complete mixing (i.e. spatially uniform $\xi$ ) occurs at $x=0.04 \mathrm{~m}$ [13]. Actually, based on the results in Fig. 7, even at $x=0.025 \mathrm{~m}$, the radial gradients of mixture fraction are very small. For $x=0.015$ and $0.02 \mathrm{~m}$, inside the IRZ, virtually complete mixing has occurred. These quick mixing 
characteristics in the chamber can also be shown in Fig. 10, where the RMS mixture fractions computed based on the filtered mixture fraction are plotted. For $x=0.005 \mathrm{~m}, 0.01 \mathrm{~m}$ and $0.015 \mathrm{~m}$ in Figs. 10(a)-10(c), considerable RMS values of mixture fraction can be observed from the experimental data, while for downstream stations (e.g. $x \geq 0.02 \mathrm{~m}$ in Figs. 10d-i), the mixture fraction RMS values are comparatively low, i.e. below 0.01 . These have been accurately predicted in the LES, although for $x=$ $0.01 \mathrm{~m}$ and $0.015 \mathrm{~m}$, the predicted results are slightly higher than the measured ones.

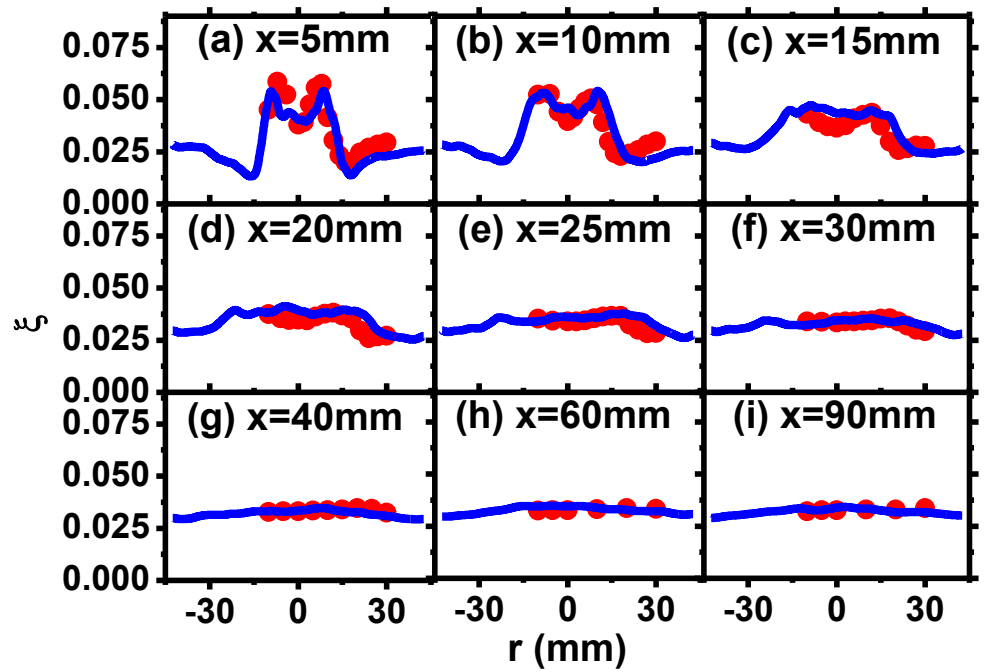

Fig. 9 Radial profiles of mean mixture fraction. Lines: LES/CMC; symbols: experimental data from Ref. [13].

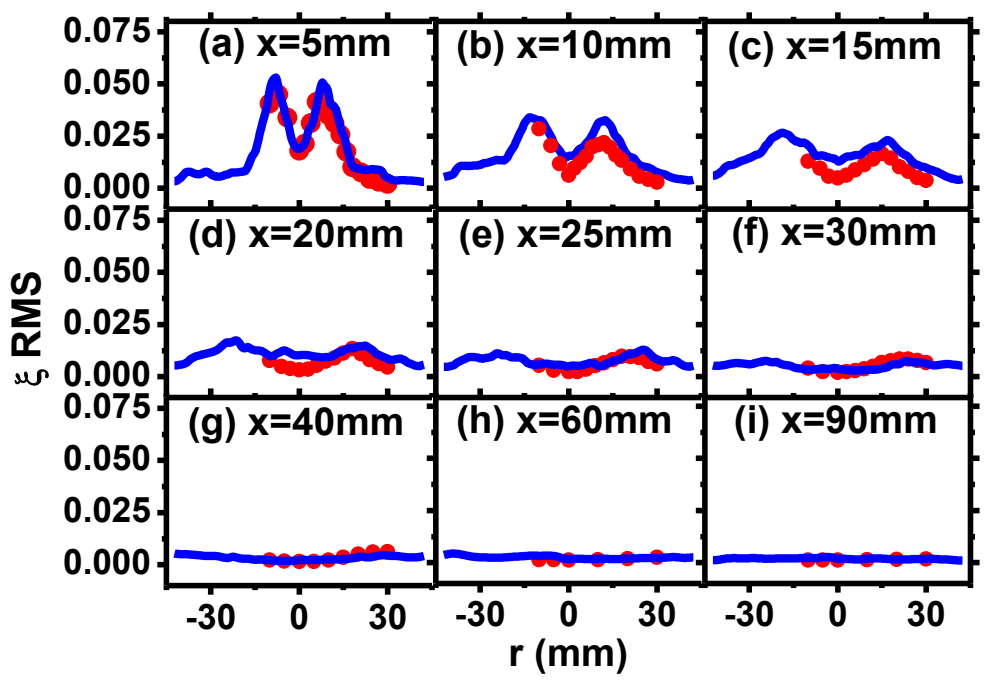

Fig. 10 Radial profiles of RMS mixture fraction. Lines: LES/CMC; symbols: experimental data from Ref. [13]. 
It is found from Fig. 11 that the simulations predict the time-averaged temperature at the upstream (e.g. $x=0.005$ and $0.01 \mathrm{~m})$ and downstream $(\mathrm{e} . \mathrm{g} . x=0.06 \mathrm{~m})$ locations reasonably well. Specifically, the general radial distributions are correctly captured, e.g. the locations of peak temperature and the radial variations of mean temperature. Also, the relative errors for the mentioned locations (e.g. $x=0.005$ and $0.01 \mathrm{~m}$ ) are below $10 \%$. However, in the stations $x=0.01-0.03 \mathrm{~m}$ in Fig. 11, over-prediction is evident, particularly for the range of $0.01 \mathrm{~m}<r<0.03 \mathrm{~m}$.

The distributions of the time-averaged mass fractions of $\mathrm{CH}_{4}, \mathrm{O}_{2}, \mathrm{CO}_{2}$ and $\mathrm{H}_{2} \mathrm{O}$ are plotted in Fig. 12. For the first location $x=0.005 \mathrm{~m}$, in IRZ (i.e. $r<0.01 \mathrm{~m}$ ) time-averaged $\mathrm{CH}_{4}$ and $\mathrm{O}_{2}$ mass fractions, $\left\langle\tilde{Y}_{C H 4}\right\rangle$ and $\left\langle\tilde{Y}_{O 2}\right\rangle$, are slightly under-predicted (for $r>0.02 \mathrm{~m}$, relative error $<15 \%$ ), while for those of $\mathrm{CO}_{2}$ and $\mathrm{H}_{2} \mathrm{O}$, slight over-prediction (for $r<0.01 \mathrm{~m}$, relative error $<5 \%$ ) can be observed. This may be due to the possibility that the flame transients near the burner exit are not accurately predicted, since we can observe the movement (e.g. frequent lift-off and flashback) of the flame base. For locations further downstream (e.g. $x=0.01$ and $0.015 \mathrm{~m}$ ), the above-mentioned under- and over-predictions persist. At $x$ $=0.04 \mathrm{~m}$ in Fig. 12 and afterwards (not shown here), the species concentration distributions become more uniform and good agreement can be seen. In general, the current LES/CMC simulations capture reasonably well the major species distributions, but there is a tendency to predict slightly more complete combustion than in the experiment in the region $0.01 \mathrm{~m}<x<0.03 \mathrm{~m}$. 


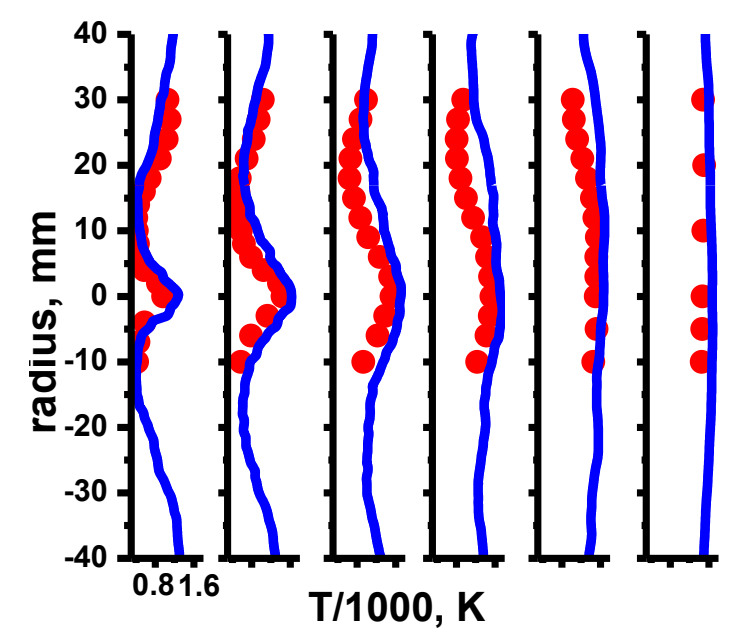

Fig. 11 Radial profiles of time-averaged temperature at (left to right) $x=5 \mathrm{~mm} ; 10 \mathrm{~mm} ; 15$ $\mathrm{mm} ; 20 \mathrm{~mm} ; 30 \mathrm{~mm}$ and $60 \mathrm{~mm}$. Lines: LES/CMC; symbols: experimental data from Ref.

[13].

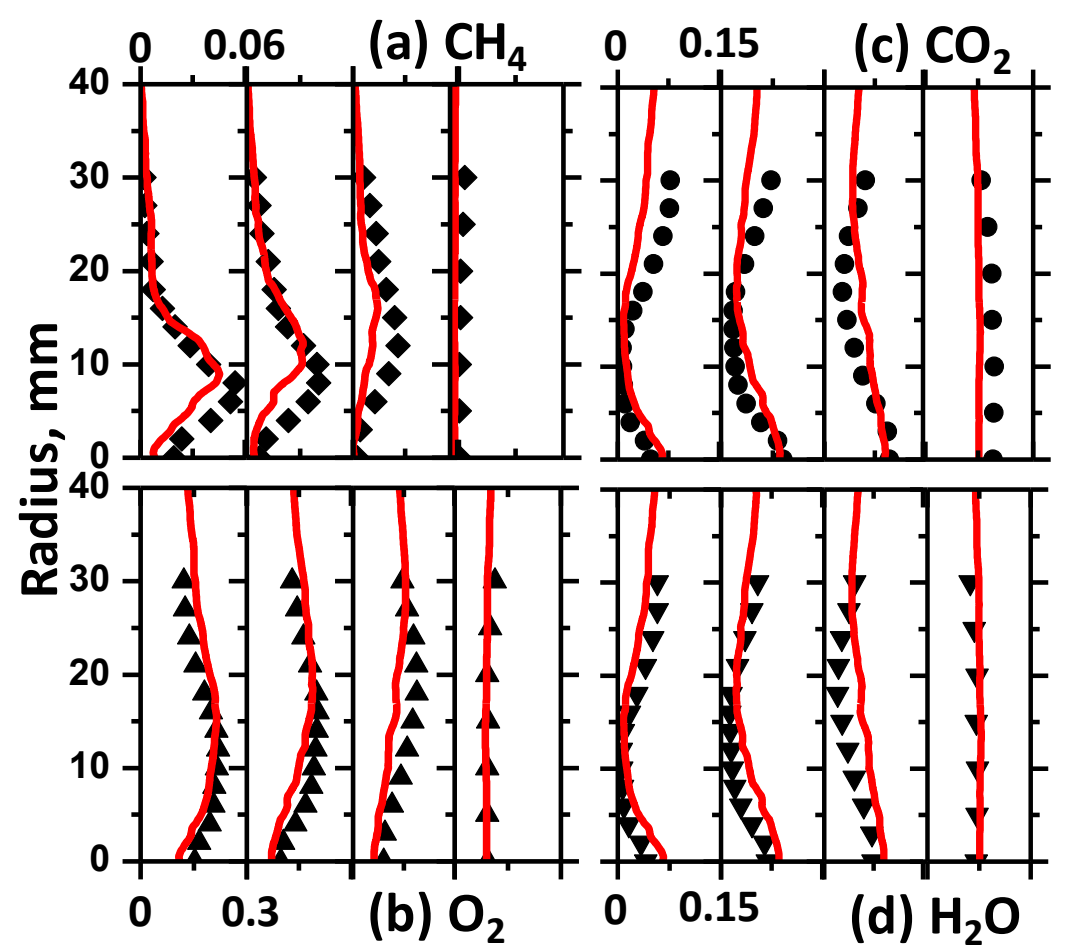

Fig. 12 Radial profiles of time-averaged mass fractions of (a) $\mathrm{CH}_{4}$, (b) $\mathrm{O}_{2}$, (c) $\mathrm{CO}_{2}$ and (d) $\mathrm{H}_{2} \mathrm{O}$. Lines: LES/CMC; symbols: experimental data from Ref. [13]. Streamwise locations for four sub-figures (left to right): $x=5,10,15$ and $40 \mathrm{~mm}$.

\subsection{Reactive scalar fields in mixture fraction space}




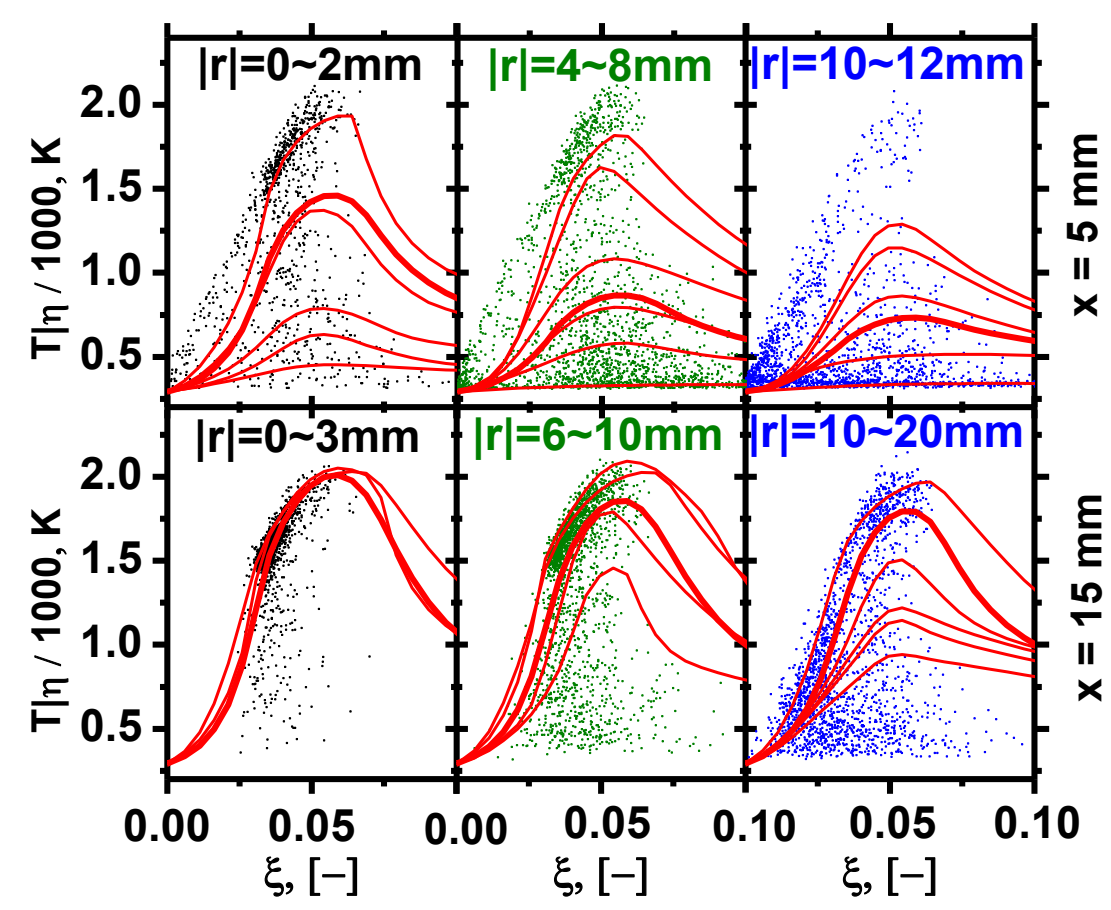

Fig. 13 Conditional temperature at two streamwise stations $x=5 \mathrm{~mm}$ (first row) and $15 \mathrm{~mm}$ (second row) for different radial sections. Thick lines: time- and space-averaged conditional temperature from LES/CMC; thin lines: instantaneous conditional filtered temperature; scatters: single-pulsed experimental data [14].

The mean (i.e. time- and space-averaged) conditional temperature $\langle\widetilde{T \mid \eta}\rangle$ computed from the statistics collection duration (i.e. $0.06 \mathrm{~s}$ ) and the respective radial sections (e.g. $|r|=0.01-0.02 \mathrm{~m}$ ), as well as some representative instantaneous $\widetilde{T \mid \eta}$, for two streamwise locations are shown in Fig. 13. The top and bottom $\widetilde{T \mid \eta}$ curves in each sub-figure roughly denote their respective fluctuation range of $\widetilde{T \eta \eta}$. For comparison, the single-shot experimental scatters from Ref. [14] for the same radial positions are added. Due to the fast mixing immediately downstream of the burner exit, only the mixture fraction range $0-0.1$ is observed. For $|r|=0-0.002 \mathrm{~m}$ at $x=0.005 \mathrm{~m}$, the experimental data span the whole shown $\xi$ range and mainly cluster around $\xi_{\mathrm{st}}=0.055$ and at high temperatures, but with a few points at intermediate values. There are also a few points with intermediate temperatures, and points corresponding to unreacted mixed fluid at various mixture fractions. These characteristics are well 
reproduced by the instantaneous solutions of the filtered CMC equation (see the thin lines). The mean conditional temperature $\langle\widetilde{T \mid \eta}\rangle$ for this location also shows this tendency, with peak temperature being about $1500 \mathrm{~K}$. A larger concentration of low-temperature samples is observed in $|r|=0.004-0.008 \mathrm{~m}$, and the simulated $\langle\widetilde{T \mid \eta}\rangle$ is lower than for $|r|=0-0.002 \mathrm{~m}$ at the corresponding mixture fraction. At $|r|=$ 0.01-0.012 $\mathrm{m}$ close to the swirling air stream, most of the points show low temperature. In LES/CMC, $\langle\widetilde{T \mid \eta}\rangle$ consistently decreases further and $\widetilde{T \mid \eta}$ fluctuates from the frozen to an intermediate solution, with no full combustion reached. At $x=0.005 \mathrm{~m}$, the conditional temperature is predicted well and the corresponding mean temperature and its fluctuation range are also reasonably well captured.

At $x=0.015 \mathrm{~m}$ and $|r|=0-0.003 \mathrm{~m}$, experimental data appear for $0.04<\xi<0.05$, most of which shows high temperature. The mean and instantaneous conditional temperature, $\langle\widetilde{T \mid \eta}\rangle$ and $\widetilde{T \mid \eta}$, agree well with the experimental data. For the radial positions of $|r|=0.006-0.01 \mathrm{~m}$, comparable experimental data are present with temperatures between $2000 \mathrm{~K}$ and $298 \mathrm{~K}$, but with a higher concentration of low temperature points. Here, $\langle\widetilde{T \mid \eta}\rangle$ is lower than that at $|r|=0-0.003 \mathrm{~m}$, but higher than that in the experiment, and the instantaneous $\widetilde{T \mid \eta}$ may drop to low values (characteristic of a local extinction $[2,5,4]$ ), but does not reach the low (unreacted) value, which is the characteristic of an unburnt-to-burnt premixed flamelet that we are probably seeing in the experiment. This also occurs for $|r|=0.01-0.02 \mathrm{~m}$, where the experimental trend of decreasing mean temperature is captured, and with the $\widetilde{T \mid \eta}$ reaching even lower values, but the frozen condition is not visited instantaneously. Therefore, the mean conditional temperature is over-predicted for most radial locations at $x=0.015 \mathrm{~m}$. This over-prediction is also observed for the time-averaged temperature in physical space, shown in Fig. 11.

Plotted in Figs. 14 and 15 are the conditional mass fractions of $\mathrm{CH}_{4}$ and $\mathrm{CO}_{2}$ for different radial sections at two streamwise locations (i.e. $x=0.005 \mathrm{~m}$ and $0.015 \mathrm{~m}$ ), respectively. The corresponding mean profiles are also added for comparison purposes. For the streamwise location $x=0.005 \mathrm{~m}$, one can 
qualitatively see the computed conditional mean $\mathrm{CH}_{4}$ and $\mathrm{CO}_{2}$ mass fractions basically reflect the tendency, i.e. most of the scatter with high and low values even around stoichiometric mixture fraction $\xi_{s t}=0.055$. Nevertheless, for $x=0.015 \mathrm{~m}$, around the stoichiometric mixture fraction, $\mathrm{CH}_{4}$ is almost depleted. However, there is a large proportion of the experimental points with high $\mathrm{CH}_{4}$ mass fraction. These observations are consistent with those made from the conditionally filtered temperature in Fig. 13. It is possible that this local over-prediction of the flame reactivity (as shown from $x=0.015 \mathrm{~m}$ in Figs. 13-15) is the reason why the present LES/CMC did not reproduce the intermittent blow-off behavior observed in this flame. Although the global flame features, and the scalars in most combustor locations, are reproduced accurately, the over-prediction of the reaction progress in some critical locations could be responsible for missing blow-off when the flame is very close to its global blow-off condition. For such conditions, the single-conditioned, first-order LES/CMC model may need extension to double conditioning or second-order closure, so the proper reaction rate is captured even in conditions with large conditional scatter as in Figs. 13-15. This is beyond the scope of this paper, but will be considered in the future. Nevertheless, it is significant to note that the present model captured most of the range of the conditional fluctuations, suggesting perhaps that a finer CMC mesh could have been sufficient. 


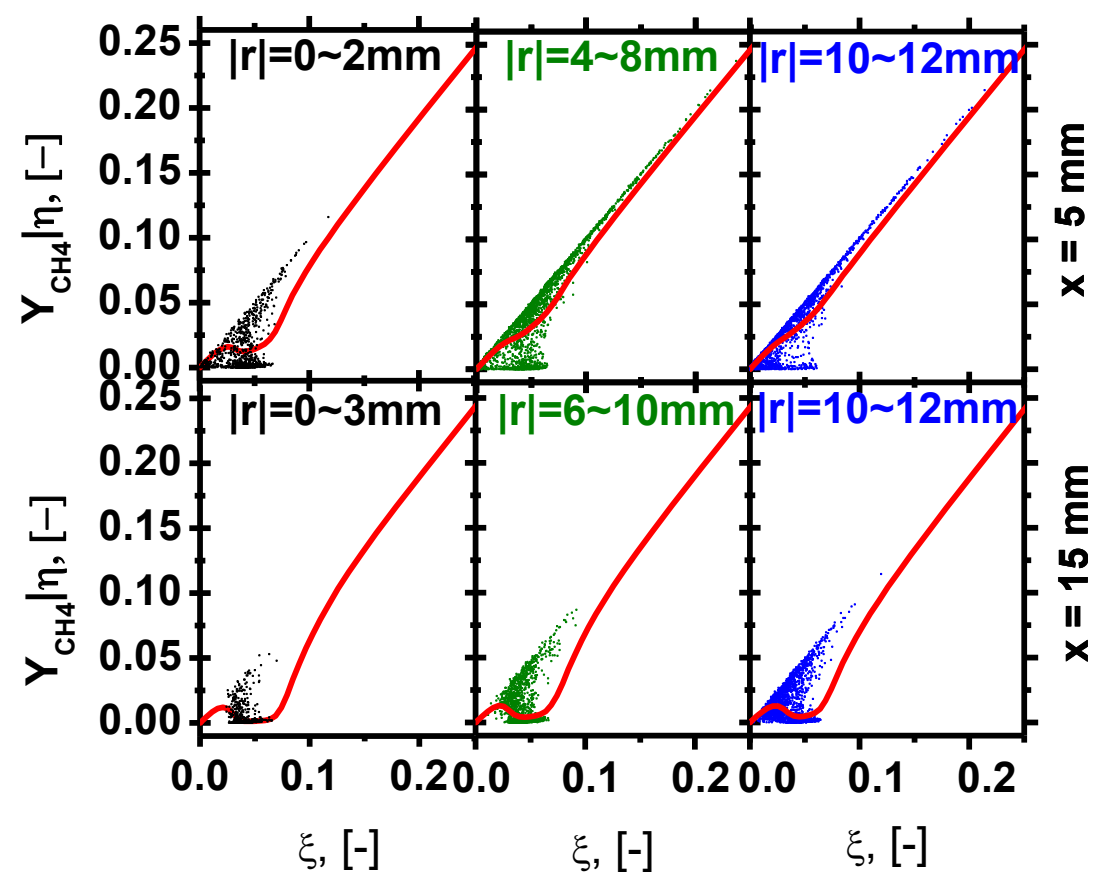

Fig. 14 Conditional $\mathrm{CH}_{4}$ mass fraction at two streamwise stations $x=5 \mathrm{~mm}$ (first row) and $15 \mathrm{~mm}$ (second row) for different radial sections. Lines: time- and space-averaged conditional temperature from LES/CMC; scatters: single-pulsed experimental data [14].

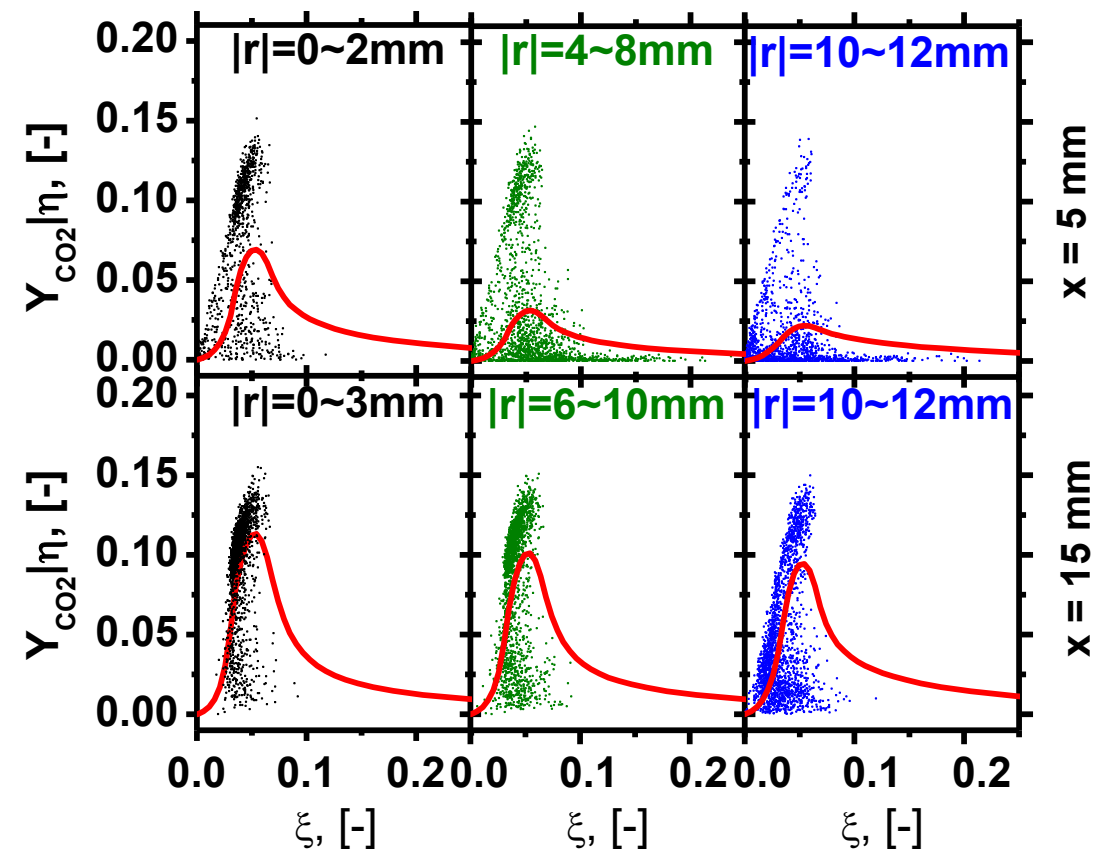

Fig. 15 Conditional $\mathrm{CO}_{2}$ mass fraction at two streamwise stations $x=5 \mathrm{~mm}$ (first row) and $15 \mathrm{~mm}$ (second row) for different radial sections. Lines: time- and space-averaged conditional temperature from LES/CMC; scatters: single-pulsed experimental data [14]. 


\subsection{Transient flame dynamics analysis}

For Flame C, intermittent lean blowout and re-ignition are observed experimentally by Stöhr et al. [19], leading to a periodic shift between stable operation and extinction in the chamber via re-ignition and blowout. The measured average duration between two neighboring stable burning events is $1-2 \mathrm{~s}$ [19], which is not affordable for LES/CMC computations considering detailed chemistry. For the time period simulated, complete flame lift-off and stabilization in the stack after quenching in the IRZ, which was highlighted in Ref. [19], was not observed. Therefore, we assume that the simulated $0.06 \mathrm{~s}$ completely sits in a stably burning event. In spite of this, we do see the transient behaviors of the flame root, such as lift-off and flash back, which are also experimentally observed in Ref. [19] and will also be discussed later in Figs. 18 and 19.

To quantify the global unsteady flame characteristics, the time series of total heat release rate $\mathbb{Q}$ is shown in Fig. 16(a), which spans 0.05 s. It is estimated from volume integration over the entire computational domain, i.e.

$$
\mathbb{Q}=\int_{\Omega^{L E S}} \tilde{\dot{q}} \mathrm{~d} V,
$$

where $\Omega^{L E S}$ represents the whole LES computational domain. The resolved heat release rate $\tilde{q}$ is

calculated from $\tilde{q}=\int_{0}^{1} \tilde{q} \widetilde{q} P(\eta) d \eta$ with $\widetilde{\dot{q} \mid \eta}=\sum_{\alpha=1}^{n} W_{\alpha} h_{\alpha} \widetilde{\omega_{\alpha} \mid \eta}$. Here $W_{\alpha}$ and $h_{\alpha}$ are the molecular weight and specific enthalpy of the $\alpha$-th species, respectively. It can be seen that total heat release rate shows pronounced regular oscillations with alternate appearance of pronounced maxima and minima. For the duration shown in Fig. 16(a), the peak value of $\mathbb{Q}$ is approximately $9.5 \mathrm{~kW}$ at $t=t_{0}+$ $0.05 \mathrm{~s}$, while the lowest $\mathbb{Q}$ is about $5.5 \mathrm{~kW}$ at $t=t_{0}+0.027 \mathrm{~s}$. For comparison, the thermal flame power $P_{t h}^{a}=7.6 \mathrm{~kW}$ estimated by the fuel mass flow rate the its LHV [14] is also added as the auxiliary line in Fig. 16(a). The time averaged total heat release rate $P_{\text {sim }}=7.2 \mathrm{~kW}$ from the computed heat release rate is slightly lower than $P_{t h}^{a}$. However, one can find that for most of the peaks shown in Fig. 16(a), the 
total heat release rate can be even higher than $P_{t h}^{a}$. This may be caused by accumulation of the injected methane in the upstream of the IRZ.

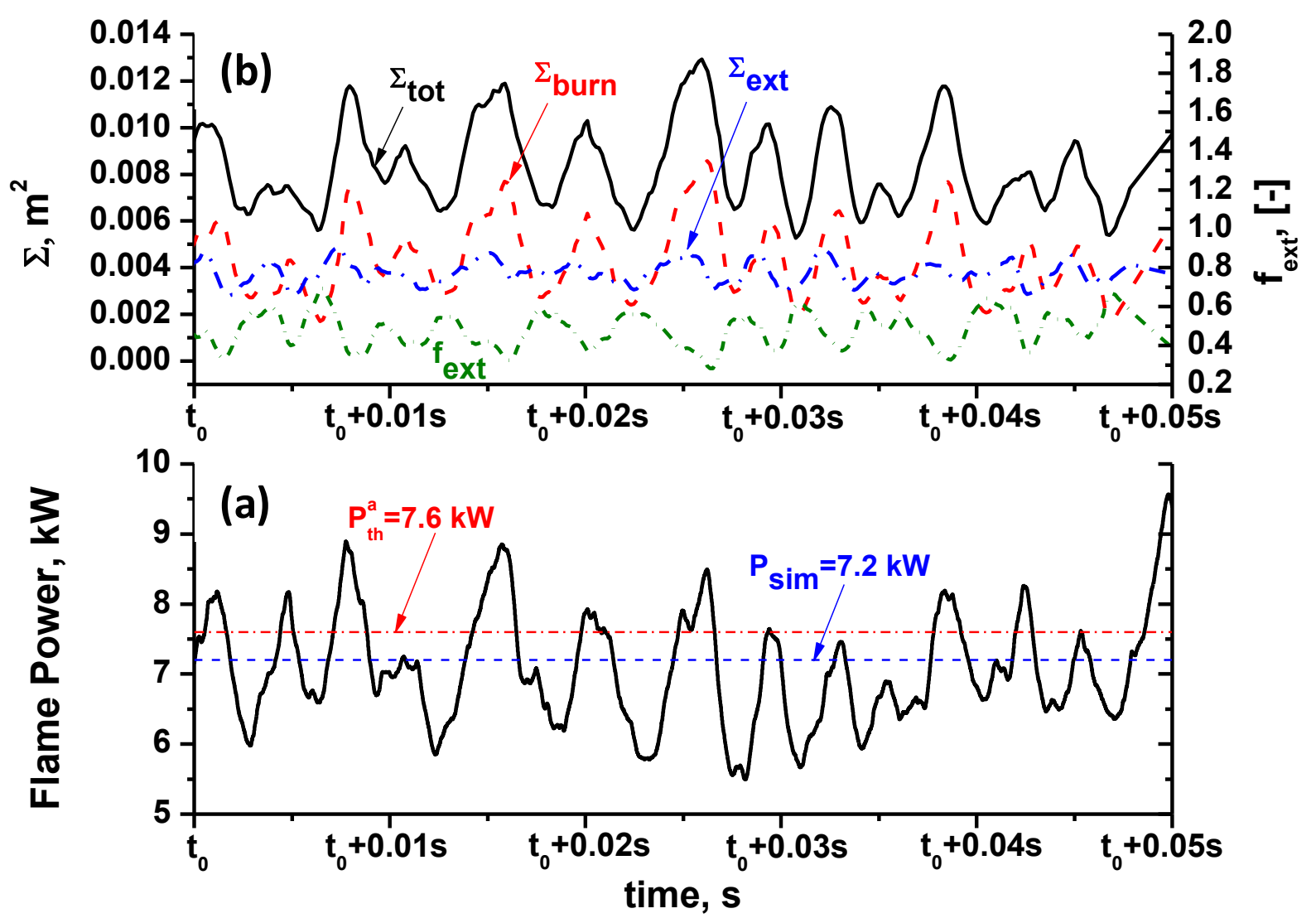

Fig. 16 Timeseries of (a) areas of stoichiometric mixture fraction iso-surface $\left(\Sigma_{\text {tot }}, \Sigma_{\text {burn }}\right.$ and $\left.\Sigma_{\text {ext }}\right)$ and extinguishing factor $\left(f_{\text {ext }}\right)$ as well as $(\mathrm{b})$ volume integrated heat release rate $(\dot{Q})$. $P_{t h}^{a}$ is flame power estimated from fuel LHV, while $P_{\text {sim }}$ is the time averaged $\dot{Q}$ based on the shown time series in (b).

Accompanied by the change of total heat release rate, we also calculate the evolution of the total flame surface $\Sigma_{\text {tot }}$ (presented in Fig. 16b), corresponding to the three-dimensional iso-surfaces of the resolved stoichiometric mixture fraction. Overall, increase/decrease of the total flame surface $\Sigma_{\text {tot }}$ follows almost identical tendency with total heat release rate in Fig. 16(a). The areas of the burning and extinguished flame surfaces, i.e. $\Sigma_{\text {burn }}$ and $\Sigma_{\text {ext }}$, are also shown in Fig. 16(b). Here we used the

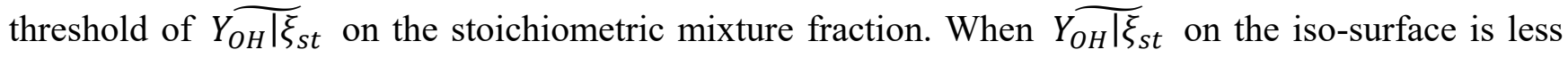


than 0.00024 (the same threshold used as that in Ref. [5]), it is burning, while ${\overline{Y_{O H} \mid \xi_{s t}}}_{>0.00024}$, it is extinguished. Meanwhile, to quantitatively predict the burning state of the stoichiometric mixture fraction surface, the extinguished fraction is calculated and defined as the area ratio of extinguished area to the total iso-surface [7], i.e.

$$
f_{\text {ext }}=\frac{\Sigma_{t o t} \mid\left(\widetilde{Y}_{O H} \mid \xi_{s t}<0.00024\right)}{\Sigma_{t o t}}
$$

Hence $f_{\text {ext }}=1$ means the complete extinction over the entire $\xi_{s t}$ iso-surface while $f_{\text {ext }}=0$ fully burning. From Fig. 16(b), one can find that change of the burning area on the $\xi_{s t}$ iso-surface is consistent with that of $\Sigma_{\text {tot }}$ and $\mathbb{Q}$, except the initial period (i.e. from $t_{0}+0.0025 \mathrm{~s}$ to $t_{0}+0.005 \mathrm{~s}$ ). Nevertheless, for the extinguished section, this tendency is not as noticeable as that for $\Sigma_{\text {burn }}$. The magnitude of $\Sigma_{\text {ext }}$ variation is comparatively small. In addition, at the trough of the $\Sigma_{\text {burn }}$ time series, it is even lower than $\Sigma_{\text {ext }}$ for the same instant. For the extinguished fraction, it fluctuates between 0.3 and 0.7, as shown in Fig. 16(b), presenting the similar periodicity to the time series of $\Sigma_{\text {tot }}$ and $\mathbb{Q}$.

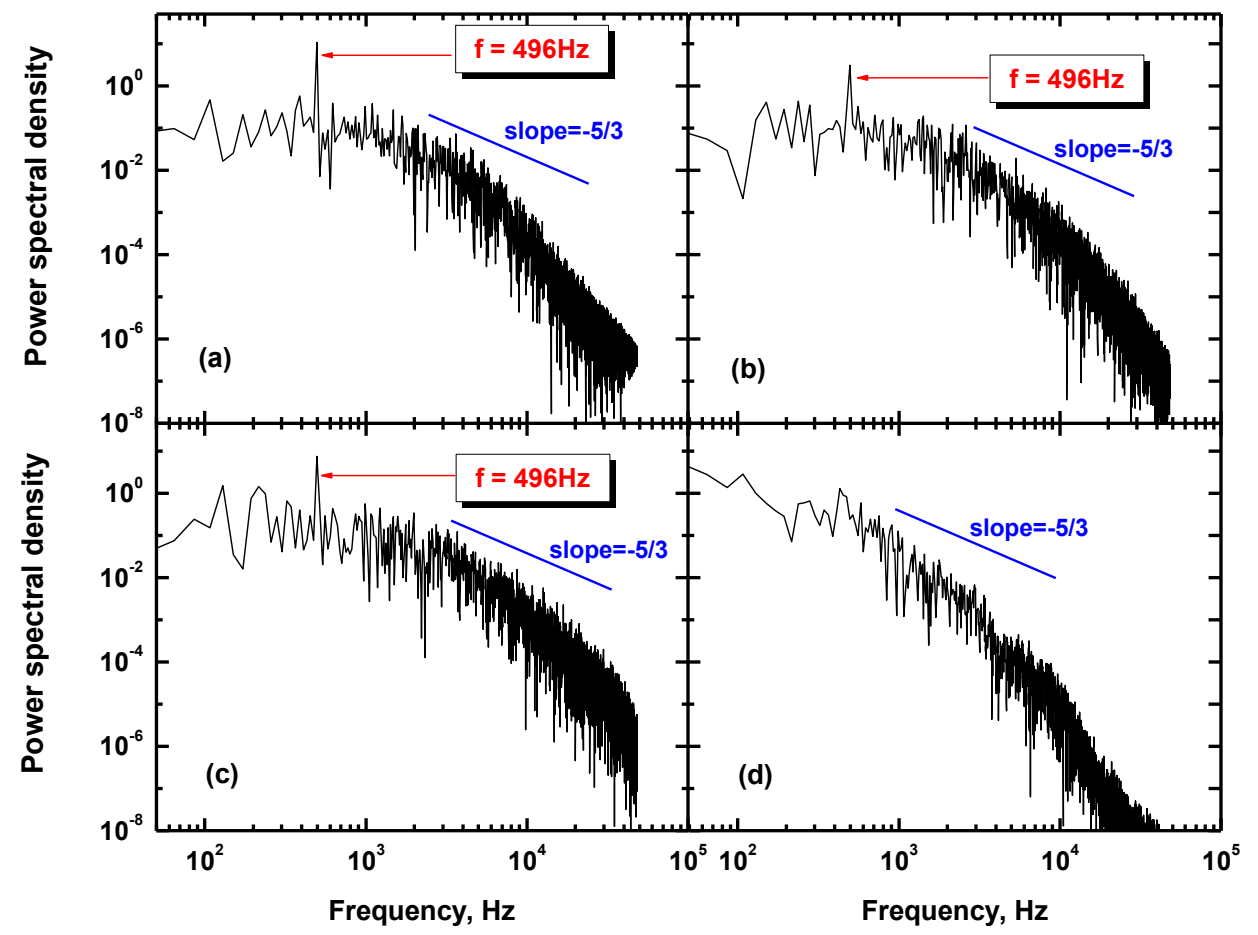

Fig. 17 Power spectral density of axial velocity as a function of frequency at various locations. The locations has been marked in Fig. 2. 
For the Flame C, it was experimentally observed that thermoacoustic combustion instability is not very strong $[13,14,19]$. In addition, low Ma number assumption is used for the current computations as mentioned in Section 2.3. Therefore, this periodic variation of the total heat release rate, presented in Fig. 16 , should not be generated by any intrinsic combustion instability for this specific flame. The timeseries of the pressure (not shown in Fig. 16) probed from one location in upstream plenum shows that the maximum pressure difference is around $50 \mathrm{~Pa}$ and there is no discernible periodic variation from the pressure time series. Therefore, one possibility for the variation of the heat release rate is an aerodynamic periodic change of the local flow field. To confirm this speculation, the PSDs (i.e. Power Spectral Density) of the axial velocity time series probed from four locations in the chamber are calculated and are shown in Fig. 17. These four probed points lie in the upstream section of IRZ close to the burner exit and loosely along the envelope of the IRZ. One can see from Figs. 17(a)-17(c) that there are pronounced PSD peaks around $f=496 \mathrm{~Hz}$. This is very close to $510 \mathrm{~Hz}$ [19], the experimentally measured frequency of precessing vortex core (abbreviated as PVC hereafter) from the Fourier analysis of velocities timeseries in IRZ. This implies the role of the PVCs in periodically amplifying and attenuating the reactivity of the swirling flame, and is also confirmed by the experimental investigations with $\mathrm{OH}^{*}$ chemiluminescence and OH-PLIF by Stöhr et al. [19]. 

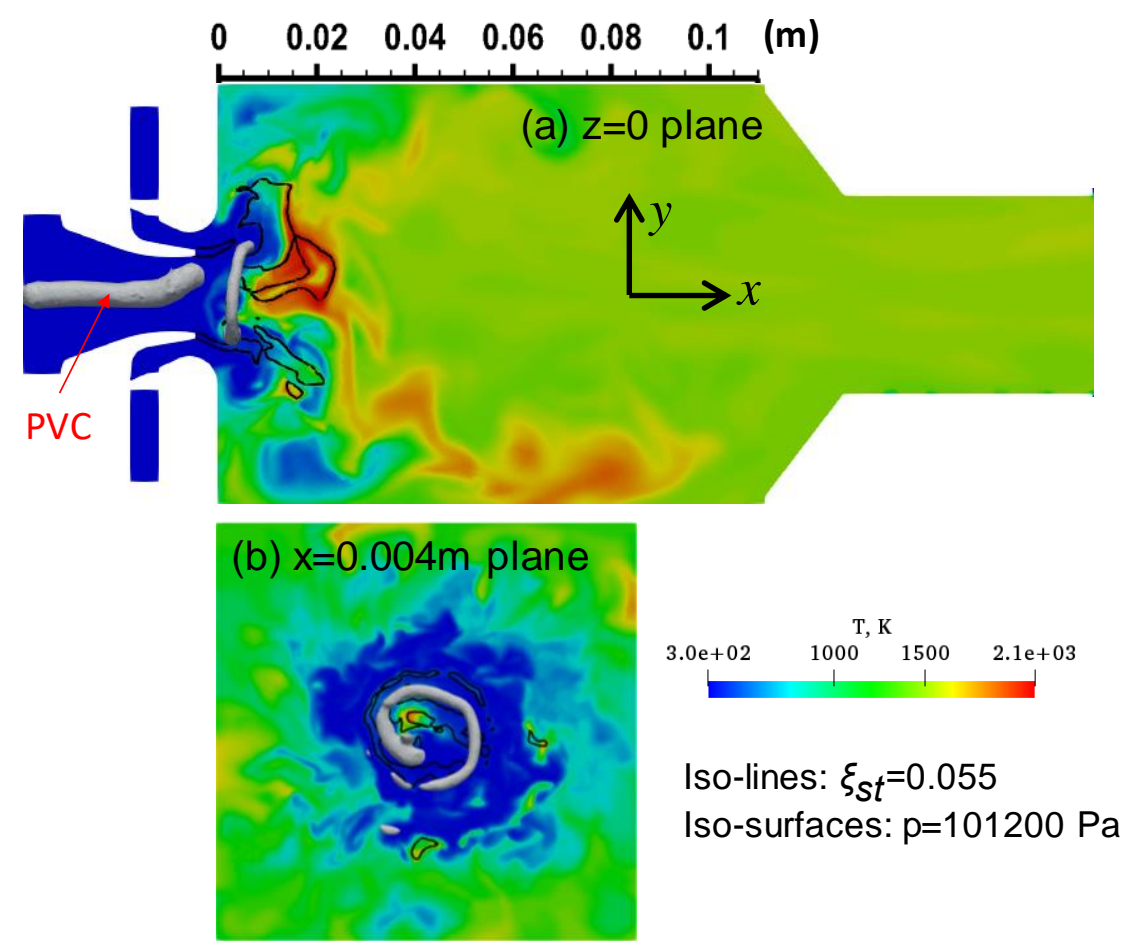

Fig. 18 PVC visualized by iso-surface of pressure ( $p=101200 \mathrm{~Pa}$ ) for (a) side view at $z=0$ plane and (b) top view at $x=0.004 \mathrm{~m}$ plane. The iso-lines are stoichiometric mixture fraction $\xi_{s t}=0.055$.

The characteristics of the PVCs in Flame $\mathrm{C}$ can be further discussed through visualizing the pressure iso-surface (i.e. $p=101200 \mathrm{~Pa}$ ) from side view and top view in Figs. 18(a) and 18(b), respectively. The PVC extends upstream until close to the inner swirler exit, and is caused by the strong local rotating motion. At $x>-0.01 \mathrm{~m}$, the PVC becomes bent, and the vortex core tends to be spanwise as shown in Figs. 18(a) and 18(b), which is located in the IRZ. This spanwise vortex is expected to play an important role in mixing the local fuel and oxidizer close to the burner exit, besides the shear layers between the inner and outer swirling flows. The PVC engulfs the finite mixture fraction (finite level of fuel/air mixing happens locally) into its center which physically acts as the flame root, thereby indicating its importance in stabilizing the swirling flame. This has also been discussed by the experimentalists recently [19], through analyzing the dynamics of flame root, helical flame and PVC. 


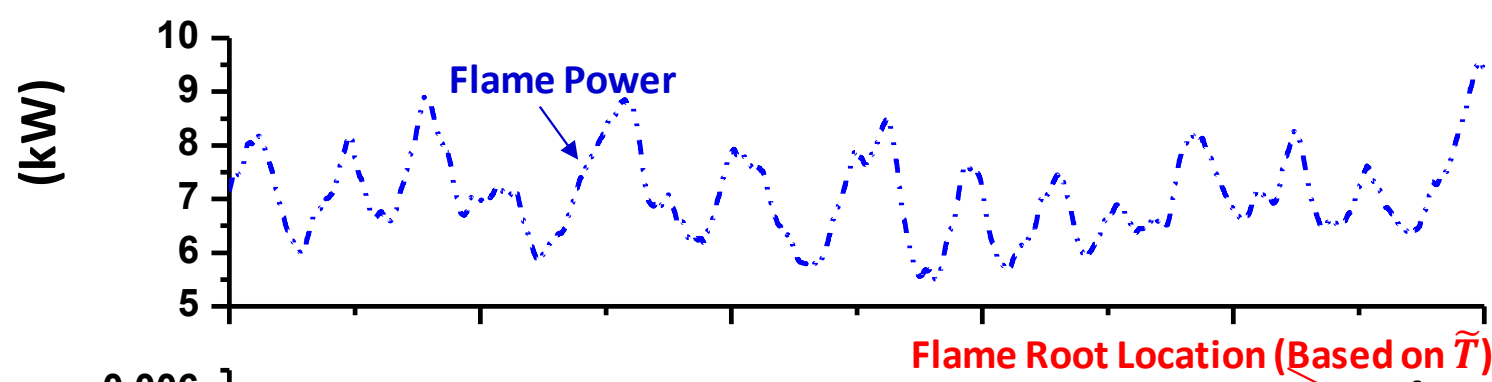

(a)

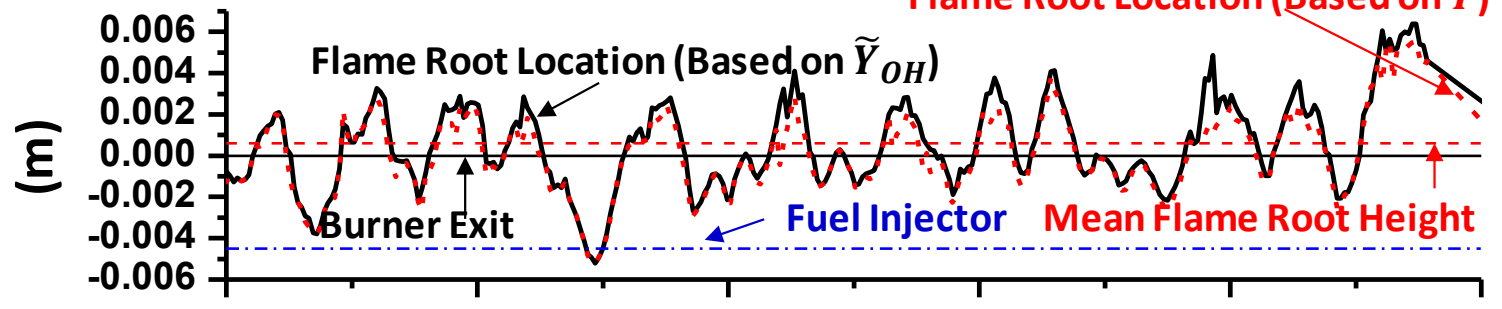

(b)

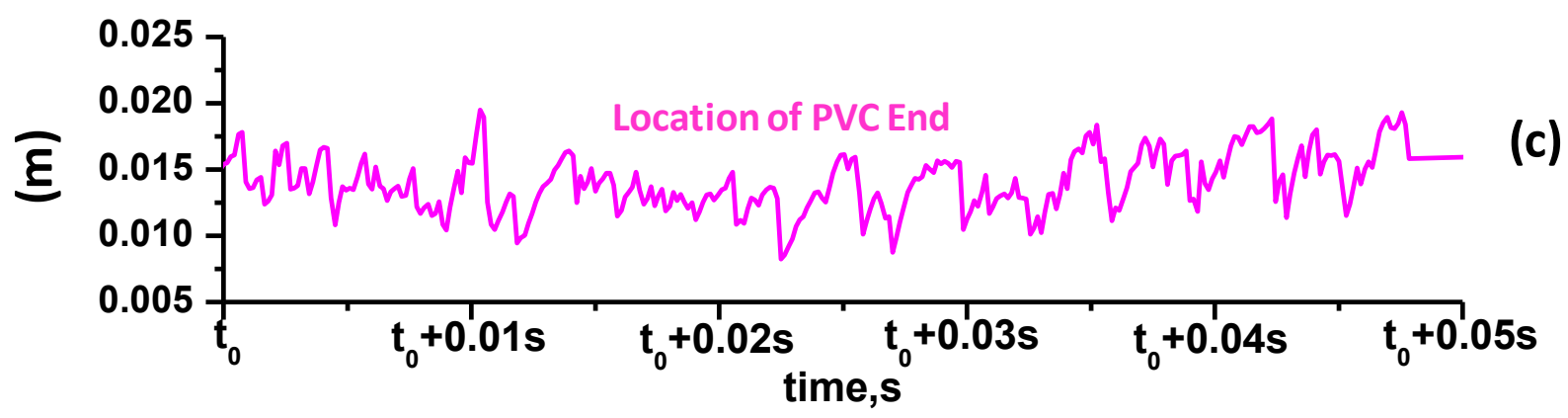

Fig. 19 Timeseries of (a) flame power, (b) flame root streamwise location and (c) streamwise location of the PVC end over the same period (from $t_{0}$ to $t_{0}+0.05 \mathrm{~s}$ ) as in Fig. 16.

The locations of the flame root are extracted from the LES/CMC simulations and plotted in Fig. 19(b) corresponding to the same period as that in Fig. 16. For reference, the timeseries of total heat release rate $\mathbb{Q}$ is also shown in Fig. 19(a), which is the same as that in Fig. 16(a). Here the flame root near the burner exit is determined from the minimum streamwise coordinate ( $x$-coordinate) where the filtered $\mathrm{OH}$ mass fraction or temperature is greater than some threshold value. Note that, from the experimentalist's point of view, the flame root locations detected using this strategy are comparable to those from line-of-sight measurements (e.g. $\mathrm{OH}^{*}$ chemiluminescence), instead of planar measurement results (e.g. OH-PLIF). As shown in Fig. 19(b), the timeseries obtained from the results of $\tilde{Y}_{O H}$ and $\tilde{T}$ are almost consistent with each other, thereby confirming the uniqueness of the flame root locations and independence on various extraction methods. The flame root periodically moves upwards and backwards, 
ranging roughly from $x=-0.005 \mathrm{~m}$ to $x=0.006 \mathrm{~m}$ as seen from Fig. 19(b). In the measurements, the statistical information of the flame root location is not reported [19]. However, it is mentioned that frequent lift-off of the flame root is observed. The averaged computed root height is $0.00061 \mathrm{~m}$ (cf. dashed line in Fig. 19b, based on flame root from $\tilde{Y}_{O H}$ ). Considerable flashback (flame root location $<$ 0 ) events can be observed and, at $t=t_{0}+0.015 \mathrm{~s}$, the flame root is even close to the fuel injectors. Additionally, when the flame propagates back, the upstream region of the IRZ also slightly moves into the central nozzle (results not shown here). Interestingly, this regular movement of the flame root shows the intrinsic correlation with the flame heat release in Fig. 19(a): lower flame root locations, exactly or with a slight phase shift, correspond to higher total heat release rate. This indicates that the thermal expansion in the combustion chamber and the flashback towards the burner exit occurs concurrently. After the flame heat release reaches its peak value, it starts to decrease gradually and flame root moves downstream and is gradually lifted off. During this process, fuel and air mixing quickly occurs around the nozzle exit. This is expected to be responsible for the next round of strong heat release from the swirling flame.

The frequency correlation of the PVCs with the heat release rate has been mentioned in Figs. 1617. Here we further examine the relation between the streamwise locations of the PVC end and the variation of the heat release rate. The locations for the downstream tip of the PVC are extracted from the maximum streamwise coordinate when the pressure is equal to $101200 \mathrm{~Pa}$ (same as that used for Fig. 18). The timeseries of these locations are demonstrated in Fig. 19(c). One can see that the PVC tip locations fluctuate between $0.01 \mathrm{~m}$ and $0.02 \mathrm{~m}$, which is qualitatively consistent with the experimental measurements (e.g. as shown in Fig. 5 of Ref. [19]). However, the correlations between the heat release rate in Fig. 19(a) and PVC end locations are not as clear as that for flame root. In spite of this, due to the continuous existence at the nozzle exit when the burner operates, its role in enhancing fuel/air mixing and stabilizing swirling flame can be confirmed, as discussed in Ref. [19]. This effect, together with the 
frequency correlation with the heat release rate fluctuation from Figs. 16 and 17, constitutes two aspects about the role of PVC in this model gas turbine combustor.

\section{Conclusions}

The LES/CMC model with detailed chemistry is applied to simulate a globally fuel-lean swirling flame in a gas turbine model combustor. The general flame shape is reasonably well captured by $\mathrm{LES} / \mathrm{CMC}$, in terms of time-averaged velocity, mixture fraction, temperature, $\mathrm{OH}$ mass fraction and heat release rate. A transient behavior of the flame root near the burner exit is found in the simulations, such as localized extinction, lift-off and flashback. A close comparison of the mean and instantaneous filtered conditional temperature showed good agreement with experimental values and trends, although in locations close to the injection point where the experiment shows a locally premixed flame behaviour, the instantaneous $\mathrm{CMC}$ solution fluctuates across a smaller range of reaction progress (i.e. do not reach the full range of unburnt-burnt seen in the experiment). Analysis of the transient flame dynamics is made, through quantifying the time series of integrated heat release rate and extinguished fraction. Periodic change of heat release rate is observed, which is accompanied by the variation of the flame root location. Lower flame root locations, exactly or with a slight phase shift, correspond to higher total heat release rate in the chamber. Overall, the LES/CMC model with single mixture fraction as the conditioning variable used in this work demonstrates satisfactory ability to capture most features of this high-mixingrate nominally non-premixed swirl-stabilized flame.

\section{Acknowledgement}

The work performed by HZ in National University of Singapore was financially supported by the start-up grant for the assistant professorship (R-265-000-604-133). The numerical simulations used UK Cirrus Cluster (http://www.cirrus.ac.uk/). Dr. Wolfgang Meier from DLR is acknowledged for sharing 
the combustor geometry and experimental data. The authors also thank the reviewers for their interesting comments to improve the manuscript.

\section{Conflict of interests}

The authors declare that they have no conflict of interest. 


\section{References}

1. Navarro-Martinez, S., Kronenburg, A., Mare, F.D.: Conditional Moment Closure for Large Eddy Simulations. Flow, Turbulence and Combustion 75(1), 245-274 (2005).

2. Garmory, A., Mastorakos, E.: Capturing localised extinction in Sandia Flame F with LES-CMC. Proceedings of the Combustion Institute 33(1), 1673-1680 (2011).

3. Garmory, A., Mastorakos, E.: Numerical simulation of oxy-fuel jet flames using unstructured LESCMC. Proceedings of the Combustion Institute 35(2), 1207-1214 (2015).

4. Zhang, H., Mastorakos, E.: Modelling local extinction in Sydney swirling non-premixed flames with LES/CMC. Proceedings of the Combustion Institute 36(2), 1669-1676 (2017).

5. Zhang, H., Garmory, A., Cavaliere, D.E., Mastorakos, E.: Large Eddy Simulation/Conditional Moment Closure modeling of swirl-stabilized non-premixed flames with local extinction. Proceedings of the Combustion Institute 35(2), 1167-1174 (2015).

6. Giusti, A., Mastorakos, E.: Detailed chemistry LES/CMC simulation of a swirling ethanol spray flame approaching blow-off. Proceedings of the Combustion Institute 36(2), 2625-2632 (2017).

7. Zhang, H., Mastorakos, E.: Prediction of Global Extinction Conditions and Dynamics in Swirling Nonpremixed Flames Using LES/CMC Modelling. Flow, Turbulence and Combustion 96(4), 863889 (2016).

8. Ukai, S., Kronenburg, A., Stein, O.T.: LES-CMC of a dilute acetone spray flame. Proceedings of the Combustion Institute 34(1), 1643-1650 (2013).

9. Ukai, S., Kronenburg, A., Stein, O.T.: Large eddy simulation of dilute acetone spray flames using CMC coupled with tabulated chemistry. Proceedings of the Combustion Institute 35(2), 16671674 (2015).

10. Bolla, M., Wright, Y.M., Boulouchos, K., Borghesi, G., Mastorakos, E.: Soot Formation Modeling of n-Heptane Sprays Under Diesel Engine Conditions Using the Conditional Moment Closure Approach. Combustion Science and Technology 185(5), 766-793 (2013).

11. Borghesi, G., Mastorakos, E., Devaud, C.B., Bilger, R.W.: Modeling evaporation effects in conditional moment closure for spray autoignition. Combustion Theory and Modelling 15(5), 725-752 (2011).

12. Wright, Y.M., De Paola, G., Boulouchos, K., Mastorakos, E.: Simulations of spray autoignition and flame establishment with two-dimensional CMC. Combustion and Flame 143(4), 402-419 (2005).

13. Meier, W., Duan, X.R., Weigand, P.: Investigations of swirl flames in a gas turbine model combustor: II. Turbulence-chemistry interactions. Combustion and Flame 144(1), 225-236 (2006).

14. Weigand, P., Meier, W., Duan, X.R., Stricker, W., Aigner, M.: Investigations of swirl flames in a gas turbine model combustor: I. Flow field, structures, temperature, and species distributions. Combustion and Flame 144(1), 205-224 (2006).

15. Mardani, A., Fazlollahi-Ghomshi, A.: Numerical Investigation of a Double-Swirled Gas Turbine Model Combustor Using a RANS Approach with Different Turbulence-Chemistry Interaction Models. Energy \& Fuels 30(8), 6764-6776 (2016).

16. See, Y.C., Ihme, M.: Large eddy simulation of a partially-premixed gas turbine model combustor. Proceedings of the Combustion Institute 35(2), 1225-1234 (2015).

17. Widenhorn, A., Noll, B., Aigner, M.: Numerical Characterization of a Gas Turbine Model Combustor. In, Berlin, Heidelberg 2010. High Performance Computing in Science and Engineering '09, pp. 179-195. Springer Berlin Heidelberg

18. Chen, Z.: Simulation of Partially Premixed Turbulent Flames. Cambridge University (2016)

19. Stöhr, M., Boxx, I., Carter, C., Meier, W.: Dynamics of lean blowout of a swirl-stabilized flame in a gas turbine model combustor. Proceedings of the Combustion Institute 33(2), 2953-2960 (2011).

20. Pera, C., Réveillon, J., Vervisch, L., Domingo, P.: Modeling subgrid scale mixture fraction variance 
in LES of evaporating spray. Combustion and Flame 146(4), 635-648 (2006).

21. Pierce, C.D., Moin, P.: A dynamic model for subgrid-scale variance and dissipation rate of a conserved scalar. Physics of Fluids 10(12), 3041-3044 (1998).

22. Triantafyllidis, A., Mastorakos, E.: Implementation Issues of the Conditional Moment Closure Model in Large Eddy Simulations. Flow, Turbulence and Combustion 84(3), 481-512 (2010).

23. O'Brien, E.E., Jiang, T.L.: The conditional dissipation rate of an initially binary scalar in homogeneous turbulence. Physics of Fluids A: Fluid Dynamics 3(12), 3121-3123 (1991).

24. See, Y.C.: Analysis of Hydrodynamic Instabilities and Combustion Dynamics in Turbulent Reacting Flows. PhD, Stanford University (2014)

25. Ramaekers, W.J.S., Tap, F.A., Boersma, B.J.: Subgrid Model Influence in Large Eddy Simulations of Non-reacting Flow in a Gas Turbine Combustor. Flow, Turbulence and Combustion 100(2), $457-$ 479 (2018).

26. Brown, P.N., Hindmarsh, A.C.: Reduced storage matrix methods in stiff ODE systems. Applied Mathematics and Computation 31, 40-91 (1989).

27. Sung, C.J., Law, C.K., Chen, J.Y.: An augmented reduced mechanism for methane oxidation with comprehensive global parametric validation. Symposium (International) on Combustion 27(1), 295-304 (1998). 